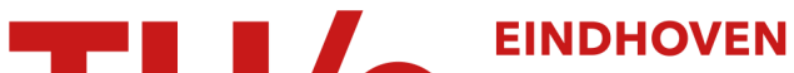 \\ UNIVERSITY OF \\ TECHNOLOGY
}

\section{Contour dynamics with non-uniform background vorticity}

Citation for published version (APA):

Vosbeek, P. W. C., Clercx, H. J. H., Heijst, van, G. J. F., \& Mattheij, R. M. M. (2000). Contour dynamics with non-uniform background vorticity. (RANA : reports on applied and numerical analysis; Vol. 0007). Technische Universiteit Eindhoven.

Document status and date:

Published: 01/01/2000

\section{Document Version:}

Publisher's PDF, also known as Version of Record (includes final page, issue and volume numbers)

\section{Please check the document version of this publication:}

- A submitted manuscript is the version of the article upon submission and before peer-review. There can be important differences between the submitted version and the official published version of record. People interested in the research are advised to contact the author for the final version of the publication, or visit the $\mathrm{DOI}$ to the publisher's website.

- The final author version and the galley proof are versions of the publication after peer review.

- The final published version features the final layout of the paper including the volume, issue and page numbers.

Link to publication

\section{General rights}

Copyright and moral rights for the publications made accessible in the public portal are retained by the authors and/or other copyright owners and it is a condition of accessing publications that users recognise and abide by the legal requirements associated with these rights.

- Users may download and print one copy of any publication from the public portal for the purpose of private study or research.

- You may not further distribute the material or use it for any profit-making activity or commercial gain

- You may freely distribute the URL identifying the publication in the public portal.

If the publication is distributed under the terms of Article $25 f a$ of the Dutch Copyright Act, indicated by the "Taverne" license above, please follow below link for the End User Agreement:

www.tue.nl/taverne

Take down policy

If you believe that this document breaches copyright please contact us at:

openaccess@tue.nl

providing details and we will investigate your claim. 


\title{
CONTOUR DYNAMICS WITH NON-UNIFORM BACKGROUND VORTICITY
}

\author{
P.W.C. Vosbeek ${ }^{\dagger \S}$, H.J.H. Clercx ${ }^{\dagger}$, G.J.F. van Heijst ${ }^{\dagger}$ \\ and R.M.M. Mattheij \\ $\dagger$ Fluid Dynamics Laboratory, ${ }^{\ddagger}$ Scientific Computing Group, \\ Eindhoven University of Technology \\ P.O. Box 513, 5600 MB Eindhoven, The Netherlands. \\ $\S$ Present affiliation: Royal Netherlands Meteorological Institute, \\ P.O. Box 201, 3730 AE De Bilt, The Netherlands, \\ E-mail: vosbeek@knmi.nl
}

In this paper it is demonstrated how a contour dynamics method can be used to simulate the behaviour of vortices in the presence of non-uniform background vorticity in general, and on the $\gamma$-plane in particular. For standard contour dynamics in case of zero or uniform background vorticity, the initial continuous vorticity distributions of the vortices are replaced by appropriate piecewise-uniform distributions. Then, the evolution of the contours separating the several regions of uniform vorticity, are followed in time. In the case of non-uniform background vorticity, it is necessary to replace the sum of the (relative) vorticity of the vortices and the background vorticity by a piecewise-uniform distribution. This has several consequences for applying the method of contour dynamics, which are discussed in this paper. The resulting method is tested on some numerical examples. One of them is (qualitatively) compared with laboratory experiments carried out in a rotating tank.

Keywords: vortex dynamics; 2D-flows; inviscid flows

\section{INTRODUCTION}

Contour dynamics is a numerical method that can be used to simulate the dynamics of twodimensional vortices. The method has originally been developed by Zabusky et al. (1979) and has been brought to full growth by the extensive work of Dritschel (1988a, 1988b, 
1989, 1993a). The method is based on the observation that, in case of an inviscid, incompressible, two-dimensional fluid flow, the evolution of a patch of uniform vorticity is fully determined by the evolution of its bounding contour because of conservation of vorticity. An approximation of a patch of distributed vorticity (like in real-life vortices) can be obtained by an appropriate piecewise-uniform distribution of several nested contours.

Contour dynamics has mainly been used to study vortices and their interactions in the presence of zero or uniform background vorticity and in the presence of shear and strain flows. Furthermore, contour dynamics has been used to study flow problems on the surface of a sphere (Dritschel, 1988a, 1993a, 1993b; Dritschel et al., 1992) without incorporating the effect of the planetary rotation. In geophysical applications, however, vortices are influenced by the non-uniform background vorticity caused by both the planetary rotation and the spherical shape of the earth. In the work presented in this paper, it is shown that contour dynamics can also be used to study this kind of problems, e.g. the evolution of vortices on a $\beta$-plane (approximation of the rotating earth at midlatitudes) or a $\gamma$-plane (approximation of the rotating earth near the poles). Though not presented here, a similar approach can be followed to study vortex dynamics on the surface of a rotating sphere.

In the presence of non-uniform background vorticity the relative vorticity is not conserved. This has severe consequences for applying contour dynamics since an initially piecewise-uniform distribution of vorticity will not remain piecewise-uniform throughout time, which is a prerequisite for applying contour dynamics (Dritschel, 1988a, 1988b, 1989, 1993a; Vosbeek, 1998; Zabusky et al., 1979) . Fortunately, the sum of the relative vorticity and the background vorticity, i.e. the absolute vorticity, is conserved, implying that it is possible to use contour dynamics also for simulating this class of flow problems. The difference with standard contour dynamics is that not the relative vorticity, but the absolute vorticity is replaced by a piecewise-uniform distribution.

A practical problem encountered by applying contour dynamics to flow problems with non-uniform background vorticity, is that simulations can become computationally too expensive to carry them out within a reasonable amount of time. The reason is that contours are now necessary both inside and outside the vortices (in contrast to problems 
with zero or uniform background vorticity where contours are necessary inside the vortices only). To accelerate the computations, the hierarchical element method developed by Vosbeek et al. (1999) is used.

This paper presents an accelerated contour dynamics method that can be used for simulating the evolution of vortices on a $\gamma$-plane. Numerical simulations are presented and one of them is qualitatively compared with a laboratory experiment carried out in a rotating tank. The remainder of the paper is organised as follows. In Section 2, a brief description of a standard contour dynamics method is given. Furthermore, the hierarchical element method used for accelerating simulations is briefly discussed. Section 3 deals with the contour dynamics method on a $\gamma$-plane. The equations of motions are considered, as well as the numerical model. In Section 4, the validity of the method is tested on two numerical experiments. The first concerns the evolution of a cyclonic monopole on a $\gamma$ plane, the second concerns a tripolar vortex on a $\gamma$-plane. The results of the latter numerical experiment are compared with a laboratory experiment. Finally, some concluding remarks are formulated in Section 5.

\section{CONTOUR DYNAMICS ACCELERATED WITH A HIERARCHICAL ELEMENT METHOD}

\subsection{Contour Dynamics with Zero or Uniform Background Vorticity}

In this section, a short description is given of a standard contour dynamics method that can be used to study 2D-flow problems of an incompressible, inviscid fluid with zero or uniform background vorticity. The governing equations of such flow problems are the Euler equation

$$
\frac{D \boldsymbol{u}}{D t}=\frac{\partial \boldsymbol{u}}{\partial t}+(\boldsymbol{u} \cdot \nabla) \boldsymbol{u}=-\frac{1}{\rho} \nabla p,
$$

expressing balance of linear momentum, and the continuity equation,

$$
\nabla \cdot \boldsymbol{u}=0
$$


expressing conservation of mass. Here, $\boldsymbol{u}=(u, v, 0)$ is the velocity vector representing the $2 \mathrm{D}$ flow, $t$ is time, $p$ the pressure, and $\rho$ the density (which is assumed to be constant) at a certain point $\boldsymbol{x}=(x, y, 0)$ in space. By introducing the stream function $\psi$ in the usual way

$$
\left\{\begin{array}{l}
u=\frac{\partial \psi}{\partial y}, \\
v=-\frac{\partial \psi}{\partial x},
\end{array}\right.
$$

and defining the vorticity vector $\boldsymbol{\omega}$ as

$$
\boldsymbol{\omega}=\nabla \times \boldsymbol{u}=(0,0, \omega),
$$

the equations of motion can be written completely in terms of the stream function $(\psi)$ and the vorticity $(\omega)$ and take the following form:

$$
\begin{aligned}
\frac{D \omega}{D t} & =\frac{\partial \omega}{\partial t}+J(\omega, \psi)=0, \\
\nabla^{2} \psi & =-\omega,
\end{aligned}
$$

where the Jacobian operator is defined as

$$
J(\omega, \psi)=\frac{\partial \omega}{\partial x} \frac{\partial \psi}{\partial y}-\frac{\partial \omega}{\partial y} \frac{\partial \psi}{\partial x} .
$$

Equation (5) expresses conservation of vorticity of a fluid particle. The solution of (6), the Poisson equation, in an infinite domain is formally given by

$$
\psi(\boldsymbol{x}, t)=-\iint_{\mathbb{R}^{2}} \omega\left(\boldsymbol{x}^{\prime}, t\right) G\left(\boldsymbol{x} ; \boldsymbol{x}^{\prime}\right) d x^{\prime} d y^{\prime},
$$

where

$$
G\left(\boldsymbol{x} ; \boldsymbol{x}^{\prime}\right)=\frac{1}{2 \pi} \ln \left\|\boldsymbol{x}-\boldsymbol{x}^{\prime}\right\|,
$$

i.e. Green's function of the Laplace operator for an infinite domain. The norm $\|\cdot\|$ is defined by $\|\boldsymbol{x}\|=\left(x^{2}+y^{2}\right)^{1 / 2}$, for each $\boldsymbol{x} \in \mathbb{R}^{2}$.

In contour dynamics, the initial continuous vorticity distribution $\omega$ of a vortex (or Fig. 1 
vortices) is replaced by a piecewise-uniform distribution $\tilde{\omega}$

$$
\tilde{\omega}(\boldsymbol{x}, 0)=\sum_{l=0}^{m} \omega_{l}, \quad \boldsymbol{x} \in \mathcal{G}_{m}(0) \backslash \mathcal{G}_{m+1}(0), \quad m=0, \ldots, M,
$$

where the regions $\mathcal{G}_{m}(0)$ are nested, $\mathcal{G}_{m+1}(0) \subset \mathcal{G}_{m}(0)$ for $m=0, \ldots, M-1, \mathcal{G}_{0}(0)=\mathbb{R}^{2}$ and $\mathcal{G}_{M+1}(0)=\varnothing$, i.e. $\mathcal{G}_{M+1}(0)$ is empty. For the moment, $\omega_{0}$ is considered to be zero, implying zero background vorticity. The $\omega_{m+1}, m=0, \ldots, M-1$, can be thought of as the jump in vorticity when moving from region $\mathcal{G}_{m}(0) \backslash \mathcal{G}_{m+1}(0)$ to $\mathcal{G}_{m+1}(0) \backslash \mathcal{G}_{m+2}(0)$ (the meaning of the notation $\mathcal{G}_{m}(0) \backslash \mathcal{G}_{m+1}(0)$ is the region $\mathcal{G}_{m}(0)$ without $\mathcal{G}_{m+1}(0)$ ). Figure 1 shows an example of regions of uniform vorticity $\mathcal{G}_{m}$ at a certain time $t, t \geqslant 0$; Figure 2 shows the corresponding piecewise-uniform distribution of vorticity.

Conservation of vorticity (5) now ensures that the piecewise-uniform distribution remains piecewise-uniform throughout time. Furthermore, it can be derived that the velocity field $\boldsymbol{u}(\boldsymbol{x}, t)$ at a certain time $t$, anywhere in the flow, and in particular on the contours $\mathcal{C}_{m}$ where $\tilde{\omega}(\boldsymbol{x}, t)$ is discontinuous, can be determined by the computation of contour integrals (Dritschel, 1988b, 1989; Vosbeek et al. 1997, 1998; Zabusky et al. 1979) :

$$
\boldsymbol{u}(\boldsymbol{x}, t)=-\sum_{m=1}^{M} \omega_{m} \oint_{\mathcal{C}_{m}(t)} G\left(\boldsymbol{x} ; \boldsymbol{x}^{\prime}\right) d \boldsymbol{x}^{\prime} .
$$

In the case of uniform background vorticity where $\omega_{0} \neq 0$ but constant, a solid body rotation, for example $\left(\omega_{0} / 2\right)(-y, x, 0)$, has to be added to the velocity field $\tilde{\boldsymbol{u}}$ in (10).

The contour integrals in (10) have to be computed numerically and the contours therefore have to be approximated by a finite, but adjustable, number of nodes. Between two subsequent nodes on a contour, linear interpolation is used to determine the contour integrals in (10). The adding and removal of nodes is based on the local curvature of the contours, minimum and maximum distance between two successive nodes and quasiuniformity of the distribution of the nodes (Vosbeek et al. 1997, 1998).

The evolution of the contours can be found by integrating the velocities, determined at the nodes on the contours, over a small time step. The time integration is carried out using second order (symplectic) midpoint rule (Sanz-Serna et al., 1994) . The reason for choosing 
this scheme is that it conserves quantities like the area and circulation of the regions of uniform vorticity better than ordinary integration methods (Vosbeek et al. 1997, 1998).

\subsection{Acceleration with a Hierarchical Element Method}

In contour dynamics, the calculation of the velocities at every node on all contours is computationally the most expensive part of the method. To accelerate the calculation of the velocities, a hierarchical element method (HEM) is used. In this section a brief description of the method by Vosbeek et al. $(1998,1999)$ is given. The method is a modification of the technique developed by Anderson (1992), which is based on the fast multipole technique (Greengard et al., 1987), but does not employ multipoles themselves. Instead, approximations based on Poisson's formula are used.

The method basically consists of two parts. The first part is based on the concept of combining the contribution of several regions of piecewise-uniform vorticity into one single computational element. To this end, the (square) computational domain is divided into a number of square boxes, which is referred to as the finest level. In this way, also the regions of uniform vorticity are divided into parts (see e.g. Figure 3b). Consider now such a part of a region of piecewise-uniform vorticity $\tilde{\omega}(r, \varphi)=\omega_{m},(r, \varphi) \in \mathcal{G}_{m}, m=m_{1}, \ldots, m_{2}$ where the regions $\mathcal{G}_{m}$ are nested as in (9) (see Figure 3a)). The computational elements, which are in fact imaginary rings that enclose a box containing a region of piecewise uniform vorticity, are then constructed as indicated in Figure 3a. With the help of the direct interaction formula (10), the velocity contributions induced by the vorticity distribution inside the box are determined at points (black dots in Figure 3a) on the imaginary ring. After this has been carried out for all boxes, the computional elements at the finest level are ready. Then one proceeds to a coarser level, by combining computational elements of the finer level into larger computional elements (i.e. larger rings that enclose four boxes) as indicated in Figure 3b. This is carried out by using Poisson integrals around the centre 
of the box which are of the form

$$
\begin{aligned}
& u_{r}(r, \varphi)=\frac{a}{2 \pi r} \int_{0}^{2 \pi} u_{r}(a, \vartheta) g\left(\frac{a}{r}, \varphi, \vartheta\right) d \vartheta, \quad r>a, \\
& u_{\varphi}(r, \varphi)=\frac{\kappa}{r}+\frac{a}{2 \pi r} \int_{0}^{2 \pi}\left(u_{\varphi}(a, \vartheta)-\frac{\kappa}{a}\right) g\left(\frac{a}{r}, \varphi, \vartheta\right) d \vartheta, \quad r>a,
\end{aligned}
$$

where $\kappa=\sum_{m=m_{1}}^{m_{2}}\left(\omega_{m} A_{m}\right) /(2 \pi), A_{m}$ is the area of $\mathcal{G}_{m}, a$ is the radius of the ring, and $g$ is defined by

$$
g(\varrho, \varphi, \vartheta)=2 \varrho \frac{\cos (\varphi-\vartheta)-\varrho}{1-2 \varrho \cos (\varphi-\vartheta)+\varrho^{2}} .
$$

After this has been carried out again for all boxes on that coarser level, one proceeds to another, yet coarser level where the same procedure is repeated, and so on. At the end of this part, a hierarchy of computational elements has been constructed.

The second part of the method concerns the organisation of the computations in such a way that the technique of combining computational elements is efficient and does not lead to inaccuracies. Far away from the evaluation point, contributions of regions are combined over large areas; regions closer to the evaluation point are combined over smaller areas as indicated in Figure 4. This figure shows an example of a hierarchical clustering of regions which is used to create an approximation of the velocities at a point in the dark grey box. The velocity contributions induced by regions in the white boxes are combined into Poisson integrals (i.e. computational elements); the velocity contributions induced by regions in the light grey and dark grey boxes is computed using the direct interaction formula (10).

The Poisson integrals are calculated using a $K$-point trapezoidal rule (the larger $K$, the more accurate the method, but also the more expensive the method). In practice, $K=17$ or $K=25$. When applying the trapezoidal rule appropriately, the numerical integration appears to be spectrally accurate. Numerical experiments have shown (Vosbeek et al., 2000) that the speed-up using this strategy is significant: the order $\mathcal{O}\left(N^{2}\right)$ behaviour (where $N$ is the total number of nodes on the contours) of the original method has been reduced to approximately $\mathcal{O}(N)$. 


\section{CONTOUR DYNAMICS WITH NON-UNIFORM BACKGROUND VORTICITY}

\subsection{Equations of Motion for a Shallow Fluid on a Rotating Sphere}

The equations of motion as given in Section 2.1 are valid in an inertial or fixed frame of reference. In geophysical flows, however, position and velocity are measured with respect to a frame of reference fixed to the surface of the earth rotating relative to an inertial frame of reference. In this case, the equation for the relative motion in the rotating frame of reference takes the following form

$$
\frac{D \boldsymbol{u}}{D t}+2 \boldsymbol{\Omega} \times \boldsymbol{u}=\frac{\partial \boldsymbol{u}}{\partial t}+(\boldsymbol{u} \cdot \nabla) \boldsymbol{u}+2 \boldsymbol{\Omega} \times \boldsymbol{u}=-\frac{1}{\rho} \nabla p-\nabla \Phi_{c},
$$

where $\Omega$ is the rotation vector (with $\Omega=\|\Omega\|=7.292 \times 10^{-5} s^{-1}$ ) and $\Phi_{c}$ the centrifugal potential. Compared to the Euler equation (1), the left hand side contains one additional term $2 \Omega \times \boldsymbol{u}$ which is the Coriolis acceleration. The right hand side contains also an additional term $\left(\nabla \Phi_{c}\right)$ which is the centrifugal force per unit mass. The continuity equation remains unaltered in a rotating frame of reference.

Now, the atmosphere and the oceans of the earth can be thought of as thin layers of only a few kilometres depth, whereas the horizontal scales of the flow are typically of the order of hundreds or even thousands of kilometres. From the continuity equation (2) it then follows that the vertical velocities are much smaller than the horizontal velocities, so that the flow can be considered approximately two-dimensional.

In order to describe the flow in the earth's atmosphere and oceans, the earth is modelled as a sphere of radius $R(R=6371 \mathrm{~km})$. Assuming that the horizontal scales are much smaller than the radius $R$, the curvature of the surface of the earth can locally be neglected and a local coordinate system $(x, y, z)$ as defined in Figure 5 may be used. The relative velocity is then given by $\boldsymbol{u}=(u, v, 0)$ and the relative vorticity vector is $\boldsymbol{\omega}=(0,0, \omega)$. The rotation vector $\Omega$ has components

$$
\boldsymbol{\Omega}=\left(\Omega_{x}, \Omega_{y}, \Omega_{z}\right)=(0, \Omega \cos \varphi, \Omega \sin \varphi)
$$

where $\varphi, \varphi \in[-\pi / 2, \pi / 2]$, is the geographic latitude as defined in Figure 5. From this it 
follows that the Coriolis acceleration is given by

$$
\begin{aligned}
2 \Omega \times \boldsymbol{u} & =2 \Omega(-v \sin \varphi, u \sin \varphi,-u \cos \varphi) \\
& =(-f v, f u,-2 \Omega u \cos \varphi),
\end{aligned}
$$

where $f=2 \Omega \sin \varphi$ is the so-called Coriolis parameter or planetary vorticity. Dimensional analysis (Pedlosky, 1987) shows that the vertical component of the Coriolis acceleration is small compared to the pressure gradient and that it can be neglected. This implies that only the vertical component of the earth rotation is dynamically active. Now, equation (14) can also be written completely in terms of vorticity $(\omega)$ and stream function $(\psi)$ (c.f. Section 2.1), yielding the scalar equation

$$
\frac{\partial \omega}{\partial t}+J(\omega, \psi)+J(f, \psi)=0
$$

or

$$
\frac{D \omega}{D t}=-J(f, \psi)
$$

Apparently, in a rotating frame of reference the vorticity $\omega$ is not materially conserved, in contrast to the situation in a fixed frame of reference (Section 2.1). However, the absolute vorticity $q$, which is defined as the sum of the (relative) vorticity $\omega$ and the planetary vorticity $f$,

$$
q=\omega+f,
$$

is materially conserved. This simply follows from equation (15) and $f$ being independent of time $t$, so that

$$
\frac{D q}{D t}=\frac{\partial q}{\partial t}+J(q, \psi)=0
$$

The relative vorticity $\omega$ being not conserved in an inviscid, incompressible flow on a rotating sphere has important consequences for the dynamics of the flow: if, for example, in a fixed frame of reference a particle $\mathcal{P}$ has vorticity $\omega$ at a certain time, at any time later the vorticity will still be $\omega$, independent of the direction in which $\mathcal{P}$ has moved. For 
example, passive particles, i.e. particles with zero vorticity, will never become active. On a rotating sphere, however, if $\mathcal{P}$ moves to the north, the planetary vorticity $f$ (not necessarily its magnitude $|f|$ ) increases, which means that $\omega$ (again, not necessarily its magnitude $|\omega|$ ) has to decrease because of conservation of absolute vorticity (c.f. (17) and (18)). In the case that $\mathcal{P}$ moves to the south, $f$ decreases so that $\omega$ has to increase. In this way, a passive particle becomes dynamically active when it is moved north- or southward by the flow. This obviously results in very different dynamics in a rotating frame of reference.

The Coriolis parameter $f$ depending on the latitude, complicates the equations of motion. As a simplification, $f$ can be expanded in a Taylor series around a fixed reference latitude $\varphi_{0}$, yielding

$$
f\left(\varphi_{0}+\delta \varphi\right)=2 \Omega\left(\sin \varphi_{0}+\delta \varphi \cos \varphi_{0}-\frac{1}{2} \delta \varphi^{2} \sin \varphi_{0}+\mathcal{O}\left(\delta \varphi^{3}\right)\right)
$$

where $\delta \varphi=\varphi-\varphi_{0}$.

By neglecting linear and higher order terms (i.e. $f$ is assumed to be a constant) the $f$-plane approximation is obtained:

$$
f=f_{0}=2 \Omega \sin \varphi_{0}
$$

Obviously in this case, the term $J(f, \psi)$ vanishes in (15) and (16), so that the equations of motion as derived in Section 2.1 (with $\omega_{0}=f_{0}$ ) are valid.

The so-called $\beta$-plane approximation is obtained by retaining the linear term and neglecting higher order terms (van Heijst, 1994). From Figure 5 it follows that $R \delta \varphi=y$, so that

$$
f(\boldsymbol{x})=f_{0}+\beta y
$$

where $\beta=2 \Omega \cos \varphi_{0} / R$. In this case, equation (15) becomes

$$
\frac{\partial \omega}{\partial t}+J(\omega, \psi)-\beta \frac{\partial \psi}{\partial x}=0 .
$$

Near the poles $\left(\varphi_{0}= \pm \pi / 2\right)$ the term $\cos \varphi_{0}$ vanishes so that the $\beta$-effect is absent. In this case, the $\mathcal{O}\left(\delta \varphi^{2}\right)$-term becomes important and

$$
f(\boldsymbol{x})=f_{0}-\gamma\left(x^{2}+y^{2}\right)
$$


with $\gamma= \pm \Omega / R^{2}$. Here, the origin of the coordinate frame $(x, y, z)$ coincides with the pole and $r=\|\boldsymbol{x}\|=R \delta \varphi$ is the radial distance to the pole. This approximation is called the $\gamma$-plane approximation (van Heijst, 1994). Now equation (15) becomes

$$
\frac{\partial \omega}{\partial t}+J(\omega, \psi)-2 \gamma\left(x \frac{\partial \psi}{\partial y}-y \frac{\partial \psi}{\partial x}\right)=0 .
$$

\subsection{The Contour Dynamics Model for a $\gamma$-Plane}

In this section it is demonstrated how contour dynamics can be employed to numerically simulate the evolution of vortices on the $\gamma$-plane. Although attention is restricted to the $\gamma$-plane here, similar procedures can be followed for simulating flows on a $\beta$-plane or even on a rotating sphere.

In Section 2.1 it has been explained how standard contour dynamics works. As was pointed out there, conservation of vorticity is essential to ensure that an initially piecewiseuniform distribution remains piecewise-uniform throughout time. Since now, on the $\gamma$ plane, the absolute vorticity $q$ as defined in (17) is conserved, an initially piecewise-uniform distribution of $q$, say $\tilde{q}$, will also remain piecewise-uniform throughout time.

Consider now, for example, the initial absolute vorticity distribution of a circular cyclonic monopole on a $\gamma$-plane as depicted in Figure 6a (where an isolated, i.e. with net Fig. 6 zero vorticity, cyclonic monopole is placed on a $\gamma$-plane) or Figure $6 \mathrm{~b}$ (where a cyclonic monopole is placed on a $\gamma$-plane). Outside the vortex, the relative vorticity $\omega$ is equal to zero. Therefore, only the planetary vorticity $f$ contributes to the absolute vorticity outside the vortex and thus the absolute vorticity is quadratic in $r=\|\boldsymbol{x}\|$ there. As in the case of the $f$-plane, the relative motion depends on the relative vorticity $\omega(\boldsymbol{x}, t)$ :

$$
\nabla^{2} \psi(\boldsymbol{x}, t)=-\omega(\boldsymbol{x}, t)=-q(\boldsymbol{x}, t)+f(\boldsymbol{x}),
$$

where $f(\boldsymbol{x})=f_{0}-\gamma\left(x^{2}+y^{2}\right)=f_{0}-\gamma r^{2}$ (see (19)) in the $\gamma$-plane approximation (note that the constant $f_{0}$ is not relevant for the dynamics of the flow as can be observed from equation $(20))$. 
Consider now the circular domain

$$
\mathcal{G}=\left\{\boldsymbol{x} \in \mathbb{R}^{2} \mid\|\boldsymbol{x}\| \leqslant R_{0}^{2}\right\}
$$

where $R_{0}$ is assumed to be large enough, so that initially there is a (bounded) region $\mathcal{B}$ inside $\mathcal{G}$ containing all non-zero relative vorticity. Outside $\mathcal{B}$ the initial relative vorticity is then equal to zero, ensuring that no relative vorticity is present near the boundary of $\mathcal{G}$ in the initial state. The meaning of this is related to accuracy aspects and is explained more in detail in the Appendix.

So in the case of cyclonic monopoles on a $\gamma$-plane, as depicted in Figure $6, R_{0}$ should be chosen such that the monopole is located completely inside $\mathcal{G}$ and sufficiently far from its boundary to ensure that the monopole and the relative vorticity created by it at later moments in time will not influence the boundary of $\mathcal{G}$ too much. It then follows that $\omega(\boldsymbol{x}, t=0)=0$ for $\boldsymbol{x}$ outside $\mathcal{G}$. Now assume that this relative vorticity remains small or even zero outside $\mathcal{G}$ for a finite time $T$, then from (21) it follows

$$
\psi(\boldsymbol{x}, t)=-\iint_{\mathcal{G}} \omega\left(\boldsymbol{x}^{\prime}, t\right) G\left(\boldsymbol{x} ; \boldsymbol{x}^{\prime}\right) d x^{\prime} d y^{\prime} .
$$

Here, $G\left(\boldsymbol{x} ; \boldsymbol{x}^{\prime}\right)=\frac{1}{2 \pi} \ln \left\|\boldsymbol{x}-\boldsymbol{x}^{\prime}\right\|$ is Green's function belonging to the infinite two-dimensional plane as defined in (8). Using $\omega=q-f$, taking the $x$ - and $y$-derivatives of $\psi$, and using the fact that $\frac{\partial G}{\partial x}=-\frac{\partial G}{\partial x^{\prime}}$ and $\frac{\partial G}{\partial y}=-\frac{\partial G}{\partial y^{\prime}}$, yields

$$
\begin{aligned}
& u=+\iint_{\mathcal{G}} q\left(\boldsymbol{x}^{\prime}, t\right) \frac{\partial G}{\partial y^{\prime}} d x^{\prime} d y^{\prime}-\iint_{\mathcal{G}} f\left(\boldsymbol{x}^{\prime}\right) \frac{\partial G}{\partial y^{\prime}} d x^{\prime} d y^{\prime}=u_{q}-u_{f}, \\
& v=-\iint_{\mathcal{G}} q\left(\boldsymbol{x}^{\prime}, t\right) \frac{\partial G}{\partial x^{\prime}} d x^{\prime} d y^{\prime}+\iint_{\mathcal{G}} f\left(\boldsymbol{x}^{\prime}\right) \frac{\partial G}{\partial x^{\prime}} d x^{\prime} d y^{\prime}=v_{q}-v_{f} .
\end{aligned}
$$

The contributions $u_{f}$ and $v_{f}$ from the background vorticity $f(\boldsymbol{x})$ to the velocities $u$ and 
$v$ can be determined analytically and are given by (Vosbeek, 1998)

$$
\begin{aligned}
& u_{f}= \begin{cases}-\left(\frac{f_{0} R_{0}^{2}}{2}-\frac{\gamma R_{0}^{4}}{4}\right) \frac{y}{x^{2}+y^{2}}, & \boldsymbol{x} \notin \mathcal{G}, \\
-\frac{f_{0}}{2} y+\frac{\gamma}{4} y\left(x^{2}+y^{2}\right), & \boldsymbol{x} \in \mathcal{G},\end{cases} \\
& v_{f}= \begin{cases}\left(\frac{f_{0} R_{0}^{2}}{2}-\frac{\gamma R_{0}^{4}}{4}\right) \frac{x}{x^{2}+y^{2}}, & \boldsymbol{x} \notin \mathcal{G}, \\
\frac{f_{0}}{2} x-\frac{\gamma}{4} x\left(x^{2}+y^{2}\right), & \boldsymbol{x} \in \mathcal{G} .\end{cases}
\end{aligned}
$$

For the determination of $\boldsymbol{u}_{q}$, the method of contour dynamics is used. For this purpose the initially continuous distribution $q$ is replaced by a piecewise-uniform distribution $\tilde{q}$

$$
\tilde{q}(\boldsymbol{x}, 0)=\sum_{l=0}^{m} q_{l}, \quad \boldsymbol{x} \in \mathcal{G}_{m}(0) \backslash \mathcal{G}_{m+1}(0), \quad m=0, \ldots, M,
$$

where the regions $\mathcal{G}_{m}(0)$ are nested such that $\mathcal{G}_{m+1}(0) \subset \mathcal{G}_{m}(0)$ for $m=0, \ldots, M-1$, $\mathcal{G}_{0}=\mathbb{R}^{2}$ and $\mathcal{G}_{M+1}(0)=\varnothing$, i.e. $\mathcal{G}_{M+1}(0)$ is empty (see also Section 2.1 ). In practice, region $\mathcal{G}_{1}$ is equal to $\mathcal{G}$ at $t=0$. Furthermore, $q_{0}$ is chosen $q_{0}=0$ so that $f=0$ outside $\mathcal{G}$. Figure 7 shows two examples of a piecewise-uniform distribution of the absolute vorticity in case of cyclonic monopoles on the $\gamma$-plane. The grey area in this figure is the circular domain $\mathcal{G}$. Because of conservation of absolute vorticity, the velocity $\tilde{\boldsymbol{u}}_{q}$ at a certain time $t$ is then given by (see also (10))

$$
\tilde{\boldsymbol{u}}_{q}(\boldsymbol{x}, t)=-\sum_{m=0}^{M} q_{m} \oint_{\mathcal{C}_{m}(t)} G\left(\boldsymbol{x} ; \boldsymbol{x}^{\prime}\right) d \boldsymbol{x}^{\prime}
$$

The relative velocity field now follows from $\tilde{\boldsymbol{u}}=\tilde{\boldsymbol{u}}_{q}-\boldsymbol{u}_{f}$ and the evolution can, just like in standard contour dynamics, be found by integrating this relative velocity over a small timestep. Note that the contour $\mathcal{C}_{0}$ should be 'far enough' from the monopole, i.e. such that $\mathcal{C}_{0}$ will not deform very much (i.e. relative to the motion of the vortex) during the simulations in order to keep the results accurate (see Appendix). 


\section{NUMERICAL EXPERIMENTS}

\subsection{The Evolution of a Cyclonic Monopole on a $\gamma$-Plane}

In this section, the method is tested on the following test problem. A circular monopole with relative vorticity distribution

$$
\omega(r)=\frac{\Gamma}{\pi R^{2}} \exp \left(-\frac{r^{2}}{R^{2}}\right),
$$

is placed on a $\gamma$-plane. A vortex with such a vorticity distribution is referred to as a Lamb vortex. The azimuthal component of the velocity field induced by a Lamb vortex is given by

$$
u_{\varphi}(r)=\frac{V R}{r}\left(1-\exp \left(-\frac{r^{2}}{R^{2}}\right)\right),
$$

where $V=\Gamma /(2 \pi R)$. In the numerical simulation the length scale $R$ is chosen to be $R=0.466$. With this particular value of $R, \omega$ is approximately zero, i.e. smaller than $1 \%$ of its maximum, for $r>1$. The strength $\Gamma$ of the vortex is chosen $\Gamma=3 \pi R^{2} / 2$. Furthermore, the initial position of the vortex is given by $\boldsymbol{x}=(2.5,-2.5)$. The value of $\gamma$ is taken as $\gamma=0.02$ and the radius of the computational domain is $R_{0}=12.0$ so that $f_{0}=\gamma R_{0}^{2}=2.88$ (i.e. $q=f=0$ at boundary of $\mathcal{G}$ ). The total number of contours is 33 , and 23 of them are used to approximate the background vorticity $f$. In Figure $8 \mathrm{a}$ the contours in the computational domain are drawn (the domain shown is $[-12,12] \times[-12,12]$ ). As can be observed, the density of contours is higher around the centre of the domain (c.f. Appendix). Figure 8b shows only a part of the computational domain $([-2,5] \times[-5,2])$ and Figure 8c shows a contour plot of the relative vorticity distribution $\omega$ in the same part of the domain.

The relative vorticity $\omega$ has been obtained by first computing the relative velocity field at the grid points of a uniform square grid. This relative velocity field is computed using the contour dynamics procedure, but instead of calculating the velocities at nodes on the contours, the velocities are determined at the grid points. Then the relative vorticity is obtained by numerical differentiation (using a central difference scheme). A square grid 
of $50 \times 50$ grid points is used for the domain $[-2,5] \times[-5,2]$. Note that the calculation of the relative vorticity is post-processing only and therefore the accuracy of it does not affect the calculation of the flow evolution.

The evolution of the absolute vorticity contours is shown in Figure 9; that of the Fig. 8 relative vorticity in Figure 10. In the latter figure, positive values of $\omega$ are represented by solid lines whereas negative values are represented by dashed lines. Contours are plotted for $\omega=-0.3,-0.2,-0.1,0.1,0.3, \ldots, 1.5$.

As can be observed from Figure 9, the (cyclonic) monopole moves northward, which roughly agrees with laboratory experiments carried out by Carnevale et al. (1991). During this process, the vortex advects fluid in the northern direction (i.e. towards the centre of the domain), resulting in a region of negative relative vorticity (see Figure 10, $t=10.0$ ). The negative vorticity becomes stronger as time proceeds and causes adjacent fluid to move in the southern direction. As a consequence, positive relative vorticity is created $(t=40.0)$. This process is the beginning of the development of a so-called Rossby wave which is observed to occur when a cyclonic monopole is placed on a $\beta$-plane (Adem, 1956; Carnevale et al., 1991; Mied et al., 1979). When the monopole has arrived more or less at the north pole, a ring of negative vorticity is surrounding it and at $t=90.0$ a tripolar structure is starting to develop. At $t=120$, this tripolar structure is nicely visible in both the absolute vorticity distribution and the relative vorticity distribution.

During the evolution, the relative vorticity of the vortex decreases. This is caused by the displacement of the vortex in the northern direction where $f$ is larger $(q=\omega+f$ is conserved, thus $\omega$ has to decrease). The decrease of the maximum of relative vorticity is shown in Figure 11. The path of the vortex, or rather the path of the point of maximum relative vorticity, is shown in Figure 12. This figure clearly reveals the northward drift of the vortex. It can also be observed that the velocity of the monopole changes during the evolution: it slows down when the vortex approaches the north pole.

For the present simulation, the deformations of the outermost contour have been examined. During the evolution, the departure $|\varepsilon|$ from the initially circular shape of the contour (c.f. Appendix) remains smaller than 0.012. The initial radius of the outermost 
contour is $r_{1}=11.52$ and the absolute vorticity jump at this contour is $\Delta q=0.22357$. With these values, it follows from the lemma in the Appendix that $|\varepsilon|$ should remain smaller than 0.021 to obtain accurate results. This apparently is the case and, moreover, it appears that the deformation of other contours remains smaller than the prescribed value as well. For example, the deformations of the eighth contour (counted from outside and whose initial radius is equal to $r_{8}=7.39$ ) are smaller than the bound required, which is 0.05, while during the simulation $|\varepsilon|<0.03$. Apparently, the computational domain could have been chosen smaller in this simulation.

\subsection{Unsteady Behaviour of Tripoles on a $\gamma$-Plane}

The unsteady behaviour of tripolar vortices on a $\gamma$-plane has already been studied by Velasco Fuentes et al. (1996). In laboratory experiments carried out in a rotating fluid, they observed an asymmetric behaviour of the tripolar structure when the vortex was created at some distance from the rotation axis. Detailed investigations revealed that this particular behaviour was caused by the so-called topographic $\gamma$-plane associated with the curved free surface. Here, this example is used to qualitatively compare contour dynamics results with results from a laboratory experiment.

The laboratory experiment presented here, is similar to that by Velasco Fuentes et al. (1996) and is carried out in a rectangular tank $(1 \mathrm{~m} \times 1.5 \mathrm{~m})$ mounted on a rotating table (see Figure 13). The tank is filled with water up to a certain height $H$, with $H$ much smaller than the horizontal dimensions of the tank. The tank is rotating steadily with angular velocity $\Omega$ yielding a (uniform) value of the Coriolis parameter $f=f_{0}=2 \Omega$. Let $U$ and $R$ be characteristic velocity and length scales, respectively, of the flow inside the tank. The Rossby number (Pedlosky, 1987) is then defined by

$$
R o=\frac{U}{2 \Omega R}=\frac{U}{f R} .
$$

If the Rossby number is small $(\ll 1)$ then the nonlinear advection terms may be neglected. As a consequence, there is a geostrophic balance in the horizontal direction and a hydrostatic balance in the vertical direction and the Taylor-Proudman theorem holds. 
This theorem shows that under these conditions the velocity cannot vary in vertical direction, implying that the motion is two-dimensional and will organise in vertical Taylor columns (Pedlosky, 1987).

The rotation of the table causes the free surface to become parabolic, as depicted in Figure 13. The fluid depth $h$ as a function of the distance $r=\|\boldsymbol{x}\|$ from the rotation axis is given by

$$
h(r)=h_{0}\left(1+\frac{f^{2} r^{2}}{8 g h_{0}}\right),
$$

where $h_{0}$ is the fluid depth at the rotation axis and $g$ the gravity acceleration. In the shallow water approximation (Pedlosky, 1987), conservation of potential vorticity is given by

$$
\frac{D}{D t}\left(\frac{\omega+f}{h}\right)=0
$$

Expression (26) can be substituted in (27) and subsequently the result can be expanded in a Taylor series around $h_{0}$. If the Rossby number is assumed to be small (which is equivalent with $\omega \ll f$ here) it then follows that

$$
\frac{D}{D t}\left(\omega-\frac{f^{3}}{8 g h_{0}}\left(x^{2}+y^{2}\right)\right)=0
$$

or

$$
\frac{\partial \omega}{\partial t}+J(\omega, \psi)-\frac{f^{3}}{4 g h_{0}}\left(x \frac{\partial \psi}{\partial y}-y \frac{\partial \psi}{\partial x}\right)=0 .
$$

Comparing this result with the $\gamma$-plane approximation (20) in Section 3.1, it can be observed that both equations are identical if

$$
\gamma=\frac{f^{3}}{8 g h_{0}}
$$

The above approximation of the effect of the parabolic surface is referred to as the topographic $\gamma$-plane (van Heijst, 1994). In this approximation shallow is equivalent to north in the $\gamma$-plane approximation. 
A tripolar vortex can be generated by creating an unstable monopole. For this purpose, a bottomless cylinder of about $0.20 \mathrm{~m}$ in diameter is placed in the tank. The liquid inside the cylinder is stirred cyclonically, i.e. in the same sense as the rotation of the table. Subsequently, the cylinder is lifted and an isolated (with net zero circulation) monopole is released. Under certain conditions, which are quite easily satisfied, the monopole becomes unstable and transforms into a tripolar structure (van Heijst et al., 1991). The vortex can be visualised by adding dye to the fluid inside the cylinder before lifting it.

In the experiment presented here, the water depth $H$ was taken equal to $H=0.165 m$ and the table was rotating with angular velocity $\Omega=0.61 \mathrm{~s}^{-1}$, yielding $f=2 \Omega=1.22 \mathrm{~s}^{-1}$. Furthermore, the water depth $h_{0}$ at the rotation axis was $h_{0}=0.16 m$. With (29) it then follows that $\gamma=0.15 \mathrm{~m}^{-2} \mathrm{~s}^{-1}$.

Figure 14 shows a sequence of images of the dye experiment in which the vortex was created at a distance of approximately $0.25 \mathrm{~m}$ from the rotation axis. The first image is made 25 seconds after lifting the cylinder. At that moment, the vortex still appears to be a monopolar vortex. After the vortex has become a tripole $(t=72 s)$, the $\gamma$-effect influences the behaviour of the vortex dramatically. The elliptically shaped core of the tripole moves away together with one of the satellites $(t=85 s)$. This structure is an asymmetric dipole that moves along a curved trajectory, resulting in a collision with the other satellite $(t=98 s)$. Subsequently, an exchange of partners takes place $(t=117 s)$ and the process is repeated although with a longer trajectory of the new dipole (and the asymmetry becomes more pronounced). In the paper by Velasco Fuentes et al. (1996), this behaviour is explained in more detail.

In the numerical simulation, an appropriate initial vorticity field is needed to initialise the computation. For this purpose, a laboratory experiment (similar to the previous dye experiment and under approximately the same conditions) has been carried out in which the flow has been seeded with small particles on the fluid's surface. The experiment has been recorded by a video camera rotating with the table (see Figure 13), and the velocity of the particles could later be obtained by using the particle tracking feature of the DigImage package (Dalziel, 1992). By interpolating these particle velocities to the points of a grid, 
the vorticity field $\omega$ could be determined.

Close inspection of the data revealed that the vortex remained nearly circular up to approximately $t=72 \mathrm{~s}$ after lifting the cylinder. At that moment, the vorticity distribution of the monopole could be fitted with the vorticity profile (Carton et al., 1989)

$$
\omega(r)=\frac{U}{R}\left(1-\frac{\alpha}{2}\left(\frac{r}{R}\right)^{\alpha}\right) \exp \left(-\left(\frac{r}{R}\right)^{\alpha}\right),
$$

with $R=0.083 \mathrm{~m}, U=0.375 \mathrm{~m} / \mathrm{s}$ and $\alpha=2.1$.

For typical length and velocity scales $R$ and $U$, respectively, the dimensionless form of (20) is given by

$$
\frac{\partial \omega}{\partial t}+(\boldsymbol{u}, \nabla \omega)-2 \gamma^{*}\left(x \frac{\partial \psi}{\partial y}-y \frac{\partial \psi}{\partial x}\right)=0 .
$$

where $\gamma^{*}$ is the dimensionless version of $\gamma$ and is related to it by

$$
\gamma^{*}=\gamma \frac{R^{3}}{U}
$$

During the experiment, viscous effects and in particular the Ekman layer at the bottom of the tank, influences the strength of the vortex to a large extent (and also the vortex size, but less dramatically). This implies that during laboratory experiments, $\gamma^{*}$ changes with time since $R$ and $U$ change with time whereas $\gamma$ remains constant. In this particular laboratory experiment, $\gamma^{*}$ varied from $\gamma^{*} \approx 0.0002$ at $t=53 \mathrm{~s}$ to $\gamma^{*} \approx 0.0003$ at $t=72 \mathrm{~s}$ and $\gamma^{*} \approx 0.008$ at $t=300 s^{\dagger}$ after lifting the cylinder.

In contour dynamics simulatons, however, $\gamma^{*}$ remains constant during the computations since viscous effects are not incorporated in the contour dynamics method (see ZavalaSansón et al., (2000) for a numerical method that does incorporate the influence of the Ekman layer). Therefore, it is not possible to make a direct comparison between a laboratory experiment and a contour dynamics simulation; only a qualitative comparison of the dynamics of the flow can be made. Thus the purpose of this comparison is to find out whether an (inviscid) contour dynamics simulation can capture some specific features of the dynamics of the flow like, for example, the unsteady behaviour of the tripole.

\footnotetext{
${ }^{\dagger}$ The value of $\gamma^{*}$ at $t=300 \mathrm{~s}$ is an estimate; the deformations of the vortex make it impossible to give an accurate value of $R$ at that moment in time.
} 
The following numerical experiment shows good qualitative agreement with the dye experiment. In this simulation, a monopole with vorticity distribution according to (30) with $R=0.47, U=0.71$ is placed on a $\gamma$-plane with $\gamma=0.02$, so that $\gamma^{*}=0.003$. The steepness parameter $\alpha$ is chosen equal to $\alpha=2.5$ to ensure that the monopole is unstable (the vorticity distribution (30) is unstable for $\alpha>2$ (Carton et al., 1989)). Furthermore, the monopole has a slightly elliptical shape (aspect ratio 1.11) to enhance the instability. The initial location of the monopole is $\boldsymbol{x}=(3.0,0.0)$. This means that the monopole is situated 3.0/0.47 $=6.4$ non-dimensional units from the centre of the domain; in the laboratory experiment, this is $0.25 / 0.083=3.0$. Thus the vortex in the numerical experiment would experience a $6.4 / 3.0=2.1$ times larger gradient in the background vorticity if the values of $\gamma *$ for both experiments were the same.

The size of the computational domain is $[-8,8] \times[-8,8]$ and the number of contours used is 43 . From these 43 contours, 24 are used to approximate the background vorticity. Fig. 15 Fig. 16 Figure 15 shows the evolution of the absolute vorticity contours at several moments in time. The domain shown is $[-0.2,4.0] \times[-1.85,2.35]$. Figure 16 shows the relative vorticity, as obtained from the relative velocity field, at the same stages in the evolution. Negative relative vorticity is again represented by dashed lines. Contours are plotted for $\omega=$ $-0.3,-0.2,0.1,0.3, \ldots, 1.5$.

In both figures, the development of an asymmetric tripolar structure can be clearly observed. At $t=49$, the core of the tripole has formed a dipolar structure together with one of the satellites, which is moving towards the other satellite. Note that, compared to the simulation of the previous section (Figure 10), far less relative vorticity is created in this simulation. Only from $t=49$ onward, some created positive relative vorticity is visible near the tail of one of the satellites.

Comparison of the two figures with the dye experiment of Figure 14 shows at least until $t=49$ a remarkable resemblance with the images of the laboratory experiments up to $t=92 s^{\ddagger}$. Apparently, the numerical method is able to simulate the dynamics of the

\footnotetext{
¥The time scales of both experiments cannot be compared due to the rapid increase of $\gamma^{*}$ in the laboratory experiment and a constant $\gamma^{*}$ in the numerical experiment.
} 
vortex very well.

\section{DISCUSSION AND CONCLUSIONS}

In this paper it has been demonstrated how contour dynamics can be used in cases of non-uniform background vorticity like in the case of a $\gamma$-plane. It is clear that this kind of problems requires a modified version of the contour dynamics method. Now, instead of replacing the continuous relative vorticity distribution inside the vortex (or vortices) by a piecewise-uniform distribution, it is necessary to replace the absolute vorticity of a domain that contains the vortex (or vortices) by an appropriate piecewise-uniform distribution. The absolute velocities at the nodes on the contours are then obtained by the standard contour dynamics procedure. To obtain the relative velocities, the velocity field induced by the non-uniform background vorticity (which can be derived analytically) has to be subtracted from the absolute velocities.

A consequence of replacing the absolute vorticity distribution by a piecewise-uniform distribution, is that the number of contours becomes rather large compared to situations with zero or uniform background vorticity. It is therefore necessary to apply an acceleration scheme in order to be able to carry out numerical simulations within a reasonable amount of time. The hierarchical element method (Vosbeek et al., 2000) is used for this purpose.

Numerical experiments have been carried out to test the method. In the first experiment, the evolution of a circular cyclonic monopole on a $\gamma$-plane is simulated. The monopole moves northward while relative vorticity is created. Since this behaviour is characteristic for the evolution of monopoles in the presence of non-uniform vorticity, this experiment shows that the method captures the dynamics of the flow rather well. Another numerical experiment, which is compared with laboratory experiments that were carried out in a rotating tank, confirms this. The experiment concerns the unsteady behaviour of a tripolar vortex on a $\gamma$-plane. The comparison with the laboratory experiments shows that the present method is able to simulate the unsteady behaviour of the tripole in a good manner. 
Although only the $\gamma$-plane is considered here, similar procedures can be followed for problems on a $\beta$-plane or on a rotating sphere. There are, however, some differences with respect to the computational domain. For the $\beta$-plane case, for example, the isolines of the background vorticity are parallel straight lines running in longitudinal direction. Since the contours should be closed, a singly-periodic computational domain can be chosen to achieve this. In latitudinal direction, a finite number of contours should be used to approximate the $\beta$-effect. Like in the $\gamma$-plane case considered here, the outermost contours (two straight lines now, instead of one circular contour in the $\gamma$-plane case) may not deform too much and similar to the $\gamma$-plane it is possible to monitor and control this during a computation. The velocity field induced by the background vorticity can also be derived analytically in the $\beta$-plane case.

\section{APPENDIX}

Accuracy aspects of the contour dynamics method on a $\gamma$-plane

This Appendix addresses accuracy aspects of the method described in this paper. The first aspect concerns the accuracy of the piecewise-uniform distribution of $q$ in the region around the vortex or vortices as an approximation to the background vorticity. Consider for this purpose a $\gamma$-plane, initially without any relative vorticity, i.e. $\omega(\boldsymbol{x}, 0) \equiv 0$ for all $\boldsymbol{x} \in \mathbb{R}^{2}$. In the exact case without any numerical approximation, nothing will happen so the relative vorticity $\omega$ will remain zero throughout time. If this problem is simulated by the contour dynamics method, then the continuous profile of the background $f$ (which is equal to $q$ in this case) is replaced by a piecewise-uniform distribution $\tilde{f}$ as shown in Figure 17a. In this figure, $f=f_{0}-\gamma r^{2}$, with $\gamma=0.02, R_{0}=10$ and $f_{0}=\gamma R_{0}^{2}$. The value of $f_{0}$ is chosen so that $f=0$ on the boundary of the domain, but this is not relevant for the computations. The jumps are chosen uniform, and equal to $\Delta f=\gamma R_{0}^{2} / M$ with $M$ the number of jumps $(M=20$ in Figure 17$)$.

The (approximate) absolute velocities at the nodes on the contours are found by the contour dynamics procedure, and the relative velocities are found by subtracting the an- 
alytical solution (23) from the absolute velocities. This procedure causes an error in the relative vorticity $\omega$, and consequently $\omega$ is not exactly zero inside the computational domain. In fact, this error in relative vorticity is given by the solid lines in Figure 17b, when the errors due to interpolation of the contours are neglected. In this figure, the difference between the continuous profile of $f$ (dashed line in Figure 17a) and the piecewise-uniform distribution $\tilde{f}$ (solid line in Figure 17a) is plotted as a function of $r$. It is clear that $|\tilde{f}-f| \leqslant \Delta f / 2$. This error in the relative vorticity will, obviously, generate an error in the relative velocity field. Since this flow problem is axisymmetric, this error has an azimuthal component only.

It can easily be derived that if the piecewise-uniform distribution

$$
\tilde{f}=\sum_{l=1}^{m} f_{l}, \quad \boldsymbol{x} \in \mathcal{G}_{m} \backslash \mathcal{G}_{m+1}, \quad m=0, \ldots, M,
$$

approximates the continuous distribution $f=\gamma R_{0}^{2}-\gamma r^{2}$, for $r \leqslant R_{0}$ where the regions $\mathcal{G}_{m}$ are given by $\mathcal{G}_{m}=\left\{(r, \varphi) \in \mathbb{R}^{2} \mid r \leqslant r_{m}\right\}$ with $R_{0}=r_{0} \geqslant r_{1}>r_{2}>r_{3}>\ldots>r_{M}, \mathcal{G}_{0}=\mathbb{R}^{2}$ and $\mathcal{G}_{M+1}=\varnothing$, then the absolute azimuthal velocity $\tilde{u}_{f \varphi}$ is given by

$$
\tilde{u}_{f \varphi}=\frac{1}{2}\left(\sum_{l=1}^{m} f_{l} r+\sum_{l=m+1}^{M} \frac{f_{l} r_{l}^{2}}{r}\right), \quad r_{m+1} \leqslant r \leqslant r_{m} .
$$

Now, like in Figure 17, the jumps $f_{m}$ are taken uniform, i.e. $f_{m}=\Delta f=\gamma R_{0}^{2} / M$. The radii $r_{m}$ are chosen such that the circulation of region $\mathcal{G}_{m}$ is equal to the circulation of the layer of the continuous distribution between the values $f=(m-1) \Delta f$ and $f=m \Delta f$ (see also Figure 18). The radii $r_{m}$ are then given by

$$
r_{m}=\sqrt{\frac{M-m+\frac{1}{2}}{M}} R_{0}
$$

Then it follows from equation (33) that

$$
\begin{aligned}
\tilde{u}_{f \varphi} & =\frac{\Delta f}{2}\left(\sum_{l=1}^{m} r+\sum_{l=m+1}^{M} \frac{r_{l}^{2}}{r}\right) \\
& =\frac{\Delta f}{2}\left(m r+\frac{(M-m)^{2} R_{0}^{2}}{2 M r}\right), \quad r_{m+1} \leqslant r \leqslant r_{m} .
\end{aligned}
$$


If $r_{m+1} \leqslant r \leqslant r_{m}$, then $r$ can be written as

$$
r(\xi)=\sqrt{\frac{M-m+\frac{1}{2}-\xi}{M}} R_{0}, \quad 0 \leqslant \xi \leqslant 1 .
$$

Note that $r_{m}=r(0)$ and $r_{m+1}=r(1)$. With this expression for $r$, it is easy to prove that the error in the azimuthal velocity component, due to the piecewise-uniform distribution of $f$ is given by

$$
E=u_{f \varphi}-\tilde{u}_{f \varphi}=\frac{(\Delta f)^{2}}{4 \gamma} \frac{\left(\xi-\frac{1}{2}\right)^{2}}{r(\xi)}, \quad r_{m+1} \leqslant r(\xi) \leqslant r_{m} .
$$

Apparently, the error in the azimuthal velocity component is of order $\mathcal{O}\left((\Delta f)^{2} / r\right)$. This may suggest that the error near $r=0$ is unbounded. Fortunately, it follows from the definition of $r(\xi)$ that $\xi=\frac{1}{2}$ and $m=M$ when $r(\xi)=0$, so the error vanishes for $r=0$. In Figure 19, the error is plotted as a function of $r$ for several values of $M$ on both a Fig. 19 linear scale (a) and a logarithmic scale (b). The $\mathcal{O}\left((\Delta f)^{2} / r\right)$ is clearly visible as well as the quadratic behaviour between two subsequent contours. Furthermore, it is also clear that by doubling the number of contours $M$, the (maximum) error becomes four times as small, since $\Delta f$ becomes twice as small.

Although the error vanishes at $r=0$, the error near the innermost contour is bigger than for larger values of $r$. Therefore, in the numerical simulations of Section $4, \Delta f$ is chosen smaller in the centre of the computational domain.

The second accuracy aspect, considered here, concerns the deformation during the simulation of the outermost contour $\mathcal{C}_{1}$. This contour is initially a circle of radius $r_{1}$ given by (34). It can be expected that, the larger $R_{0}$ is, the smaller the deformations of this contour are and consequently the more accurate the results become. However, for larger values of $R_{0}$, the number of contours increases, thus requiring much more computational time. So, the question is for which minimum value of $R_{0}$, the results are accurate enough, i.e. the errors caused by the deformation of the outermost contour are sufficiently smaller than the overall error caused by the spatial discretization. 
This question is very hard to answer, since the influence of the vortices within $\mathcal{G}$ and, moreover, the influence of the relative vorticity created by them, on the outermost contour can hardly be estimated in advance. It is, however, possible to give an a priori bound for the relative deformations, so that during the simulations it can be checked whether $R_{0}$ was chosen large enough. It might even be possible to enlarge the domain by adding one or more contours during a computation if that would be necessary. However, this option is currently not implemented in the present computer code.

Assume that during a computation, the outermost contour of the computational domain is deformed in such a way that it can be described by

$$
r(t, \varphi)=r_{1}(1+\varepsilon(\varphi, t)), \quad 0 \leqslant \varphi<2 \pi, \quad 0 \leqslant t \leqslant T,
$$

where $r$ is the radial distance to the centre of the domain, $\varphi$ is the azimuthal angle with respect to the positive $x$-axis and $\varepsilon$ is a function of $\varphi$ and time $t$ and is assumed to be small, i.e. $|\varepsilon(\varphi, t)| \ll 1$ for $0 \leqslant t \leqslant T$. The following lemma shows how small $|\varepsilon|$ and thus the relative deformations of the outermost contour actually should be in order to ensure sufficiently accurate results.

Lemma If the disturbance of the outer contour can be described by (38) and

$$
|\varepsilon(\varphi, t)| \leqslant-1+\sqrt{1+\frac{\Delta q}{2|\gamma| r_{1}^{2}}}=\frac{\Delta q}{4|\gamma| r_{1}^{2}}+\mathcal{O}\left(\left(\frac{\Delta q}{|\gamma| r_{1}^{2}}\right)^{2}\right),
$$

then

$$
|\omega(r(t, \varphi), \varphi, t)| \leqslant \frac{\Delta q}{2},
$$

where $\Delta q$ is the jump in absolute vorticity near the boundary of the computational domain. Proof. The proof simply follows from conservation of absolute vorticity. Since $\omega$ is assumed to be zero on the outer contour at $t=0$, conservation of absolute vorticity yields

$$
f_{0}-\gamma r_{1}^{2}=\omega(r(t, \varphi), \varphi, t)+f_{0}-\gamma r_{1}^{2}(1+\varepsilon(\varphi, t))^{2} .
$$


From this it follows that

$$
\omega(r(t, \varphi), \varphi, t)=\gamma r_{1}^{2}\left(2 \varepsilon(\varphi, t)+\varepsilon^{2}(\varphi, t)\right)
$$

Using (39) simply yields

$$
\begin{aligned}
|\omega(r(t, \varphi), \varphi, t)| & \leqslant|\gamma| r_{1}^{2}\left(2|\varepsilon(\varphi, t)|+\varepsilon^{2}(\varphi, t)\right) \\
& \leqslant|\gamma| r_{1}^{2} \frac{\Delta q}{2|\gamma| r_{1}^{2}} \\
& =\frac{\Delta q}{2}
\end{aligned}
$$

This lemma states, that if $\varepsilon$ satisfies (39) during a simulation, then the relative vorticity generated by the perturbation of the outer contour remains smaller than the error in the relative vorticity caused by the piecewise-uniform distribution discussed previously. In the case of uniform jumps in the absolute vorticity, $\Delta q$ is given by $\Delta q=\gamma R_{0}^{2} / M$ with $M$ the number of jumps necessary to approximate the background $f$ accurately.

\section{REFERENCES}

Adem, J. (1956) A series solution for the barotropic vorticity equation and its application in the study of atmospheric vortices, Tellus, VIII 364-372.

Anderson, C.R. (1992) An implementation of the fast multipole method without multipoles, SIAM J. Sci. Stat. Comput., 13 923-947.

Carnevale, G.F., Kloosterziel, R.C. and van Heijst, G.J.F. (1991) Propagation of barotropic vortices over topography in a rotating tank, J. Fluid Mech., 233 119-139.

Carton, X.J., Flierl, G.R. and Polvani, L.M. (1989) The generation of tripoles from unstable axisymmetric isolated vortex structures, Europhys. Lett., 9 339-344.

Dalziel, S. (1992) DigImage, Image Processing for Fluid Dynamics, Technical report, Cambridge Environmental Research Consultants Ltd. 
Dritschel, D.G. (1988a) Contour dynamics/surgery on the sphere, J. Comput. Phys., 79 $477-483$.

Dritschel, D.G. (1988b) Contour Surgery: A Topological Reconnection Scheme for Extended Integrations Using Contour Dynamics, J. Comput. Phys., 77 240-266.

Dritschel, D.G. (1989) Contour dynamics and contour surgery: Numerical algorithms for extended, high-resolution modelling of vortex dynamics in two-dimensional, inviscid, incompressible flows, Comput. Phys. Rep., 10 77-146.

Dritschel, D.G. (1993a) A fast contour dynamics method for many-vortex calculations in two-dimensional flows, Phys. Fluids A, 5 173-186.

Dritschel, D.G. (1993b) Moment accelerated contour surgery, in J. T. Beale et al., ed., Vortex Flows and Related Numerical Methods, Kluwer Academic Publishers.

Dritschel, D.G. and Polvani, L.M. (1992) The roll-up of vorticity strips on the surface of a sphere, J. Fluid Mech., 234 47-69.

Greengard, L. and Rokhlin, V. (1987) A fast algorithm for particle simulations, J. Comput. Phys., 73 325-348.

van Heijst, G.J.F. (1994) Topography effects on vortices in a rotating fluid, Meccanica, 29 $431-451$.

van Heijst, G.J.F., Kloosterziel, R.C. and Williams, C.W.M. (1991) Laboratory experiments on the tripolar vortex in a rotating fluid, J. Fluid. Mech., 225 301-331.

Mied, R.P. and Lindemann, G.R. (1979) The propagation and evolution of cyclonic Gulf Stream rings, J. Phys. Oceanogr., 9 1183-1206.

Pedlosky, J. (1987) Geophysical Fluid Dynamics, Springer Verlag.

Sanz-Serna, J.M. and Calvo, M.P. (1994) Numerical Hamiltonian Problems, Chapman \& Hall. 
Velasco Fuentes, O.U., van Heijst, G.J.F. and van Lipzig, N.P.M. (1996) Unsteady behaviour of a topography-modulated tripole, J. Fluid Mech., 307 11-41.

Vosbeek, P.W.C. (1998) Contour Dynamics and Applications to $2 D$ Vortices, Ph.D. thesis, Eindhoven University of Technology, Eindhoven.

Vosbeek, P.W.C., Clercx, H.J.H. and Mattheij, R.M.M. (2000) Acceleration of Contour Dynamics Simulations with a Hierarchical Element Method, J. Comput. Phys., in press.

Vosbeek, P.W.C. and Mattheij, R.M.M. (1997) Contour dynamics with symplectic time integration, J. Comput. Phys., 133 222-234.

Zabusky, N.J., Hughes, M.H. and Roberts, K.V. (1979) Contour dynamics for the Euler equations in two dimensions, J. Comput. Phys., 30 96-106.

Zavala Sansón, L. and van Heijst, G.J.F. (2000) Nonlinear Ekman effects in rotating barotropic flows, J. Fluid Mech., in press. 


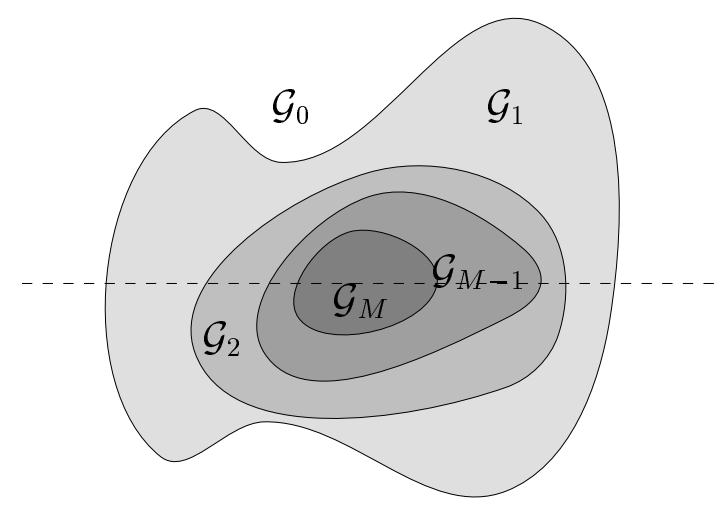

Figure 1: An arbitrary patch of piecewiseuniform vorticity distribution at a certain time $t, t \geqslant 0$. The regions $\mathcal{G}_{m}$ are nested, i.e. $\mathcal{G}_{m+1} \subset \mathcal{G}_{m}$ for $m=0, \ldots, M-1$.

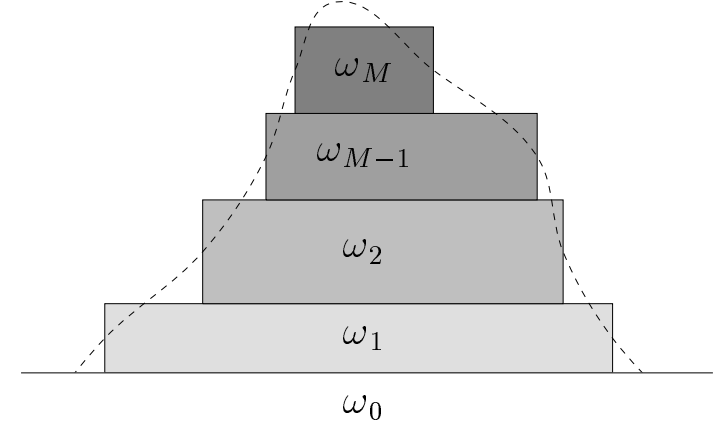

Figure 2: A cross-section (along the dashed line in Figure 1) of the piecewise-uniform vorticity profile approximating the continuous profile (dashed line). 


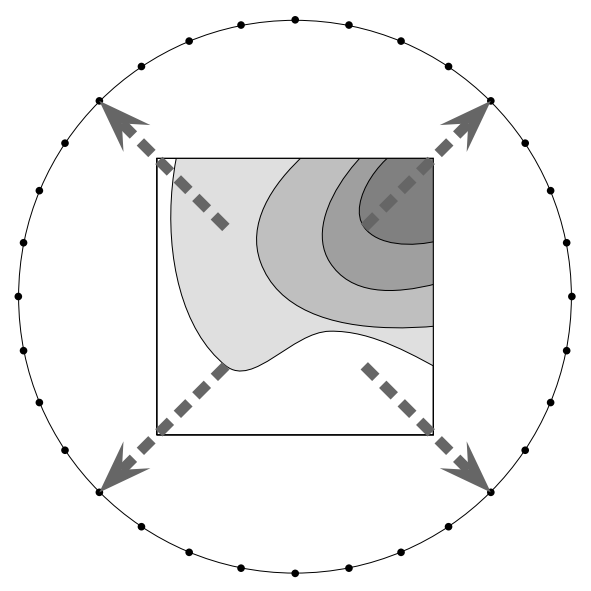

a)

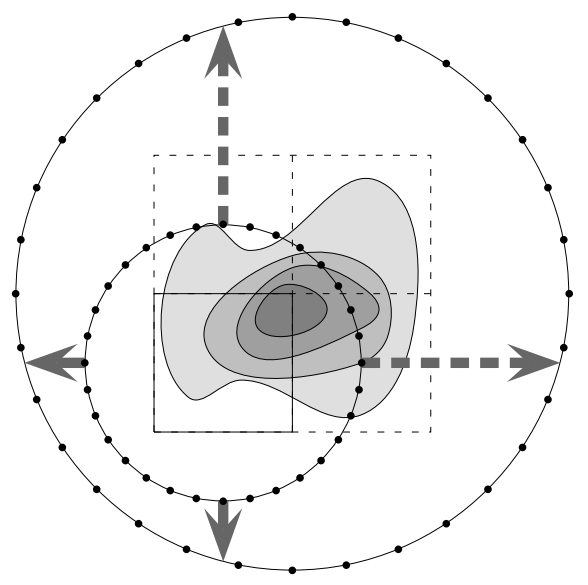

b)

Figure 3: Construction of the computational elements at the finest level using the direct interaction formula (a), and at coarser levels from the computational elements at the previous finer level (b). The shaded area's are regions of uniform vorticity; integration points at the rings are denoted with black dots. The boxes indicated with dashed lines in (b) contribute in a similar way to the large ring as the boxes drawn with a solid line. 


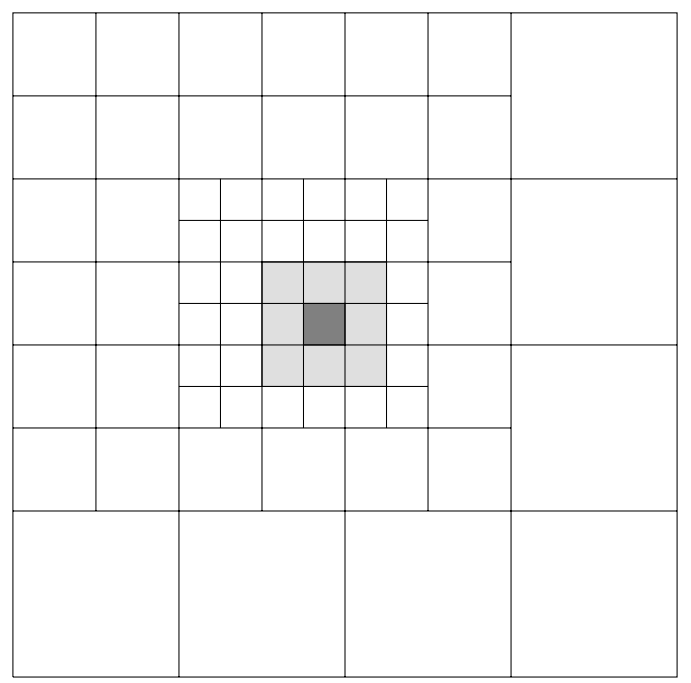

Figure 4: A hierarchical clustering of particles which is used to create a multipole approximation to the potential at a point in the dark grey box (adapted from (Anderson, 1992)). 


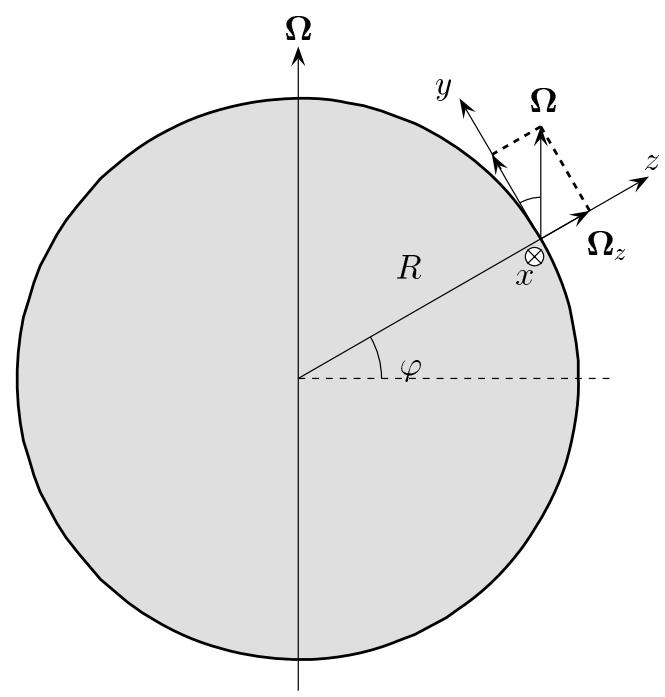

Figure 5: Definition sketch of coordinates on a rotating sphere. 


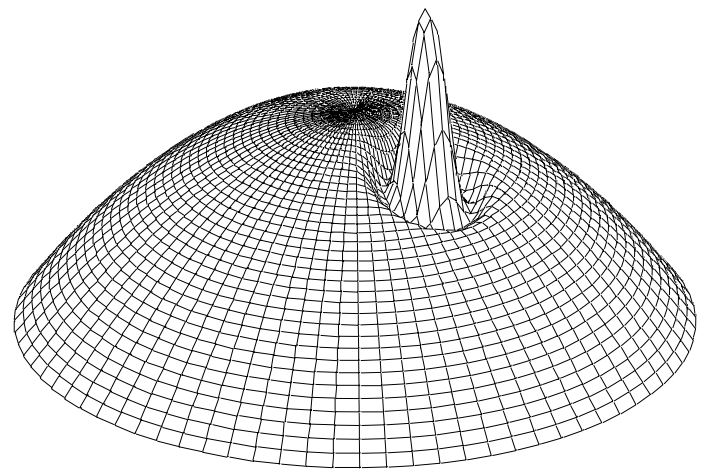

a)

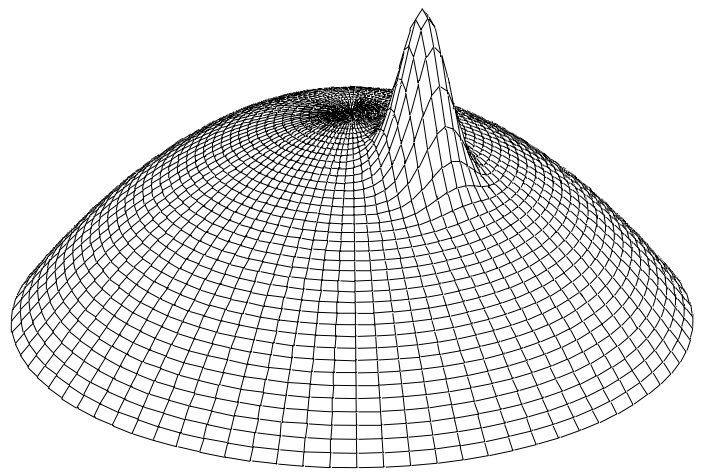

b)

Figure 6: The absolute vorticity $q$ in the case of an isolated (a) and a non-isolated (b) cyclonic monopole on a $\gamma$-plane. 


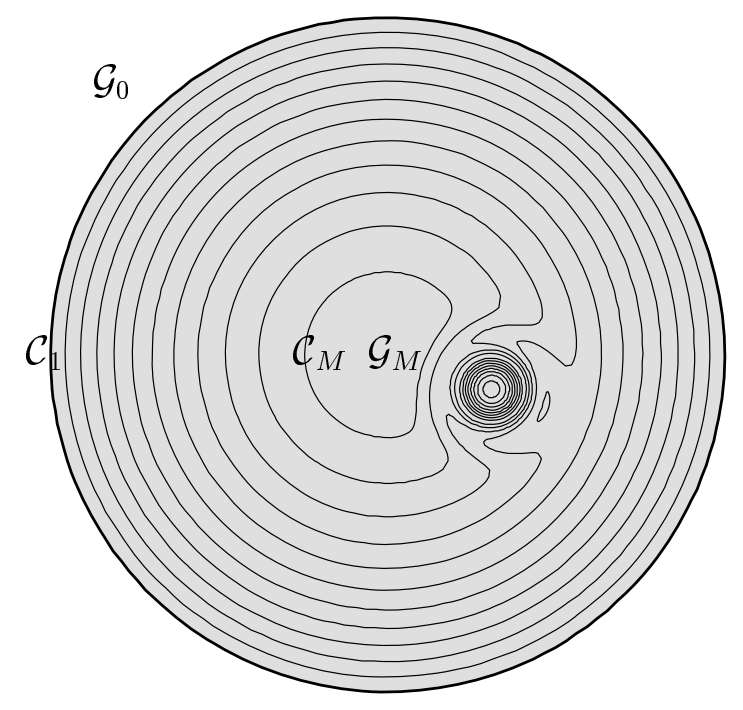

a)

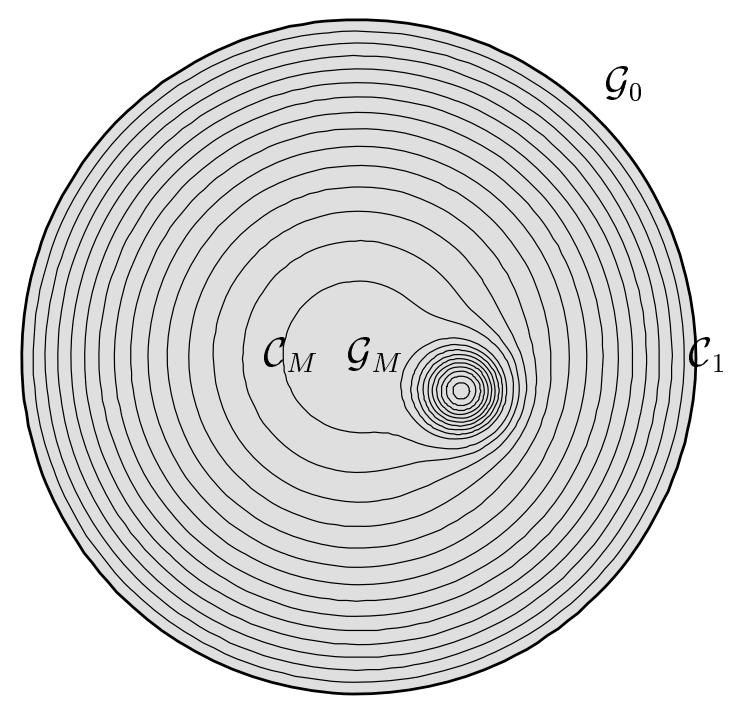

b)

Figure 7: Piecewise-uniform distribution of the absolute vorticity in the case of an isolated (a) and a non-isolated (b) cyclonic monopole on the $\gamma$-plane. $\mathcal{G}$ is the grey area in this figure. 


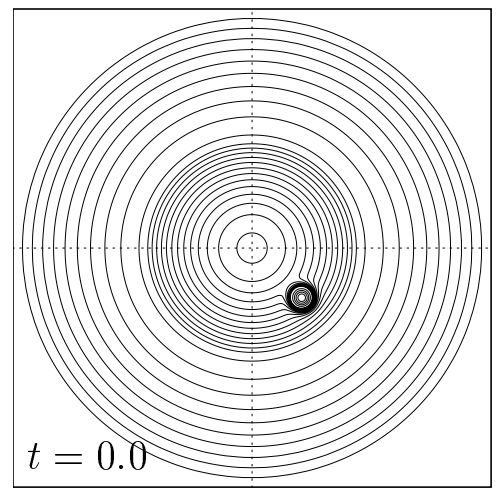

a) $[-12,12] \times[-12,12]$

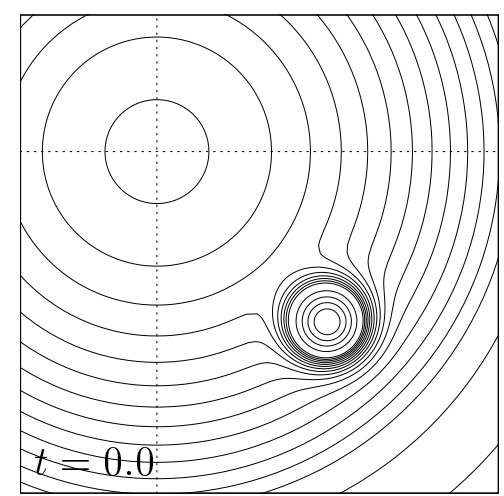

b) $[-2,5] \times[-5,2]$

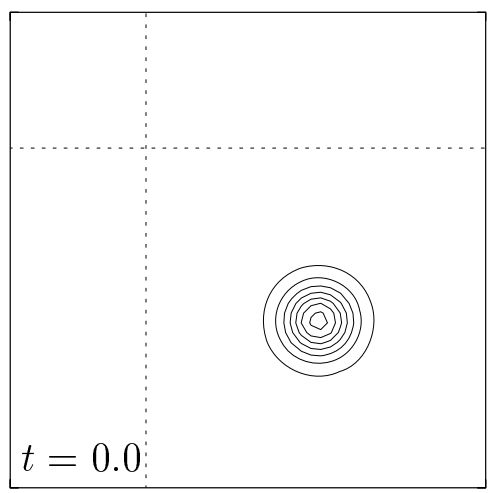

c) $[-2,5] \times[-5,2]$

Figure 8: Three plots showing the initial situation of the Lamb monopole on the $\gamma$-plane. Plot (a) shows the absolute vorticity contours in the whole computational domain, while (b) and (c) show the absolute vorticity distribution and the relative vorticity distribution, respectively, in only a part of the computational domain. 

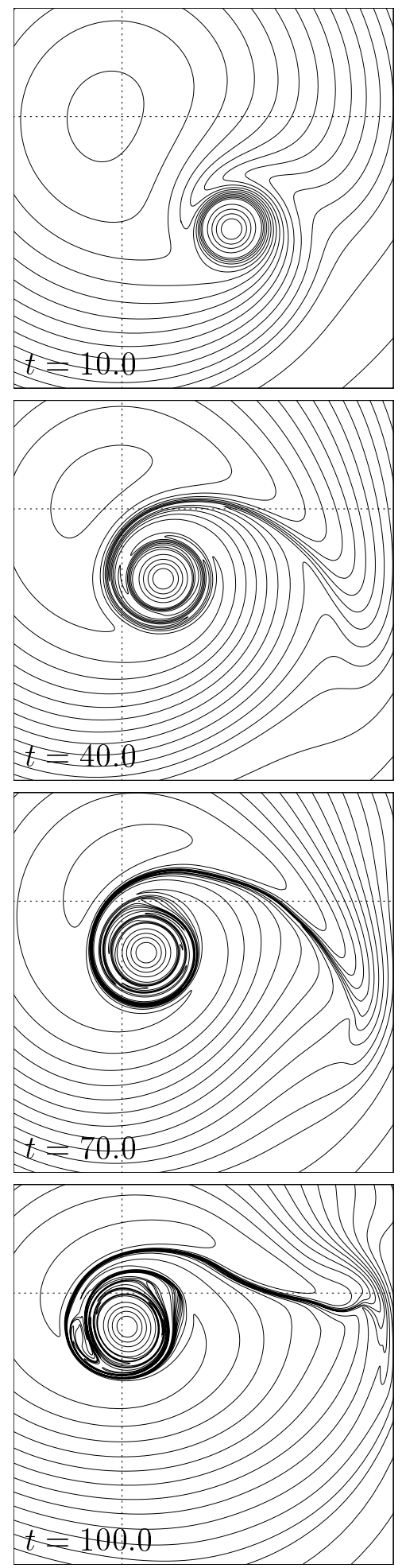
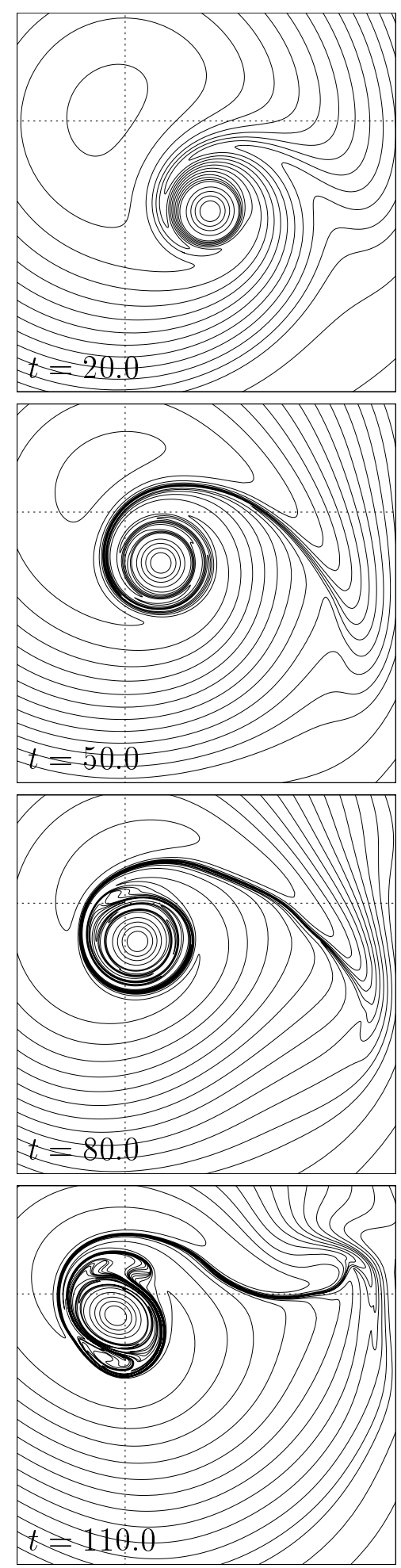
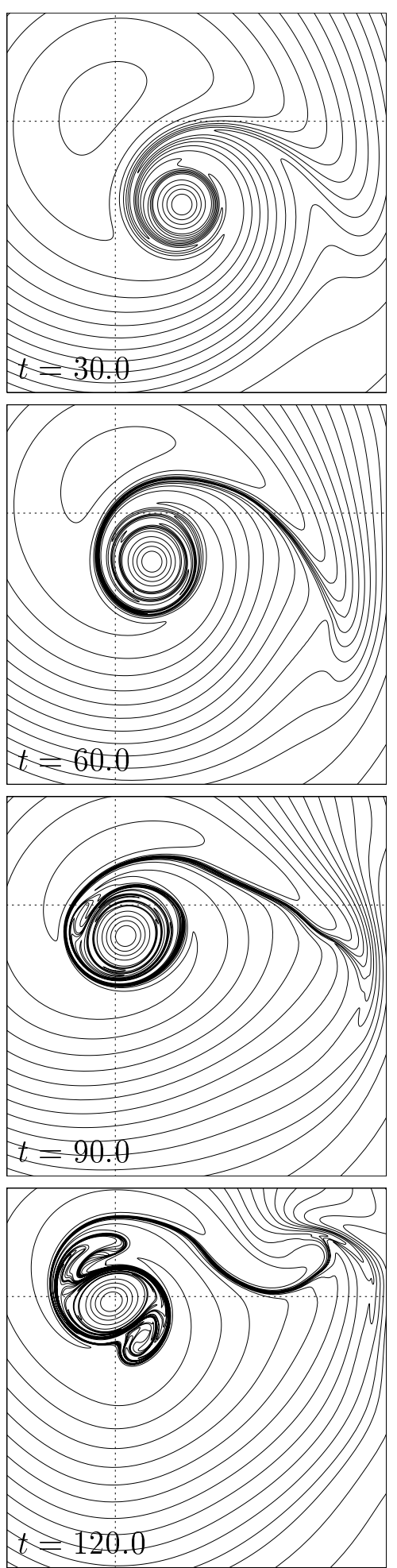

Figure 9: Absolute vorticity contours of the cyclonic vortex on the $\gamma$-plane at several moments in time. The domain shown is $[-2,5] \times[-5,2]$. 

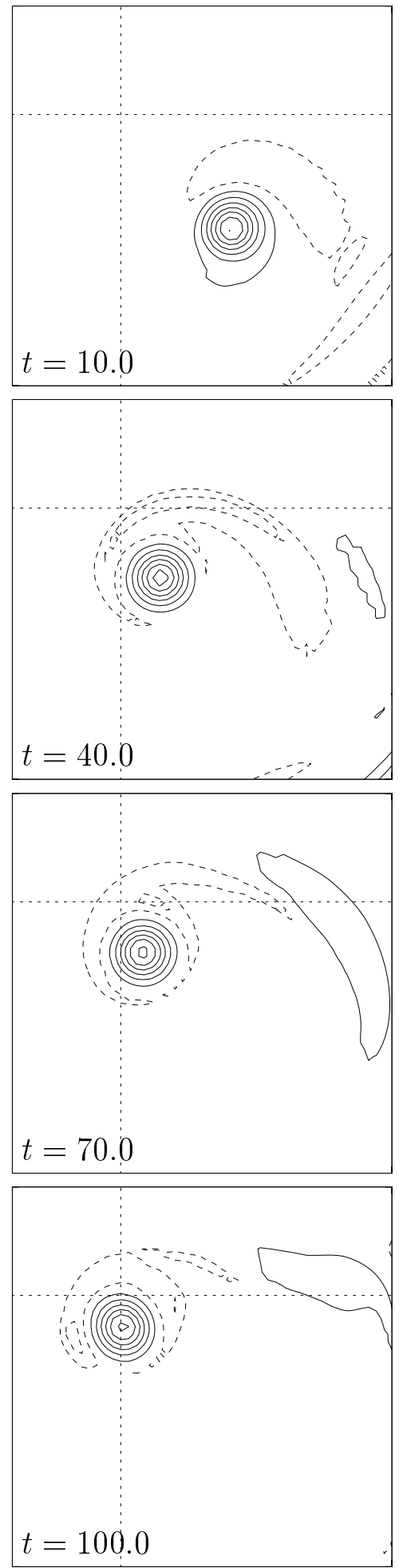
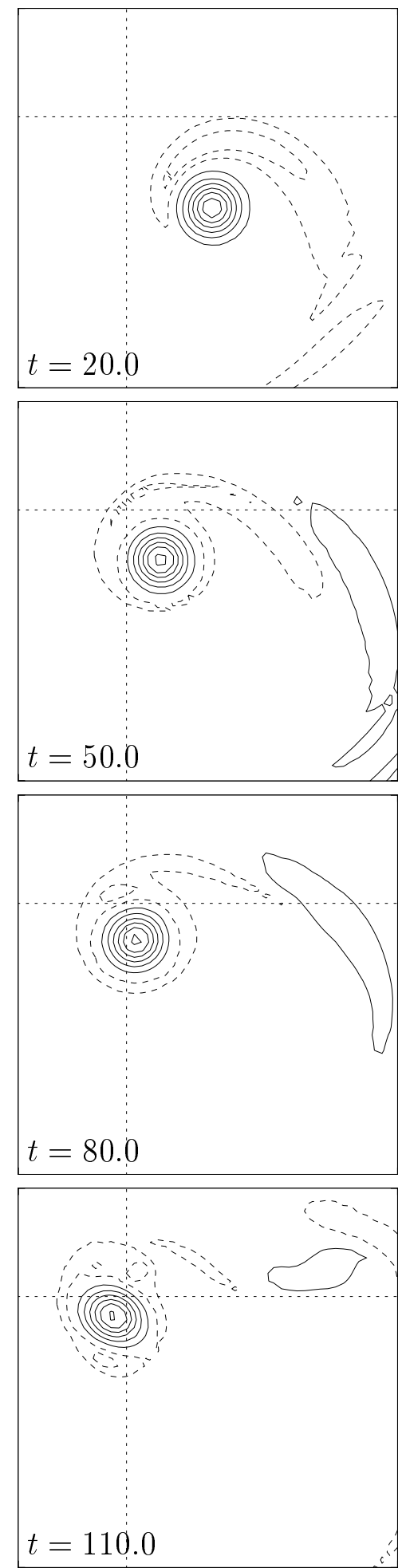
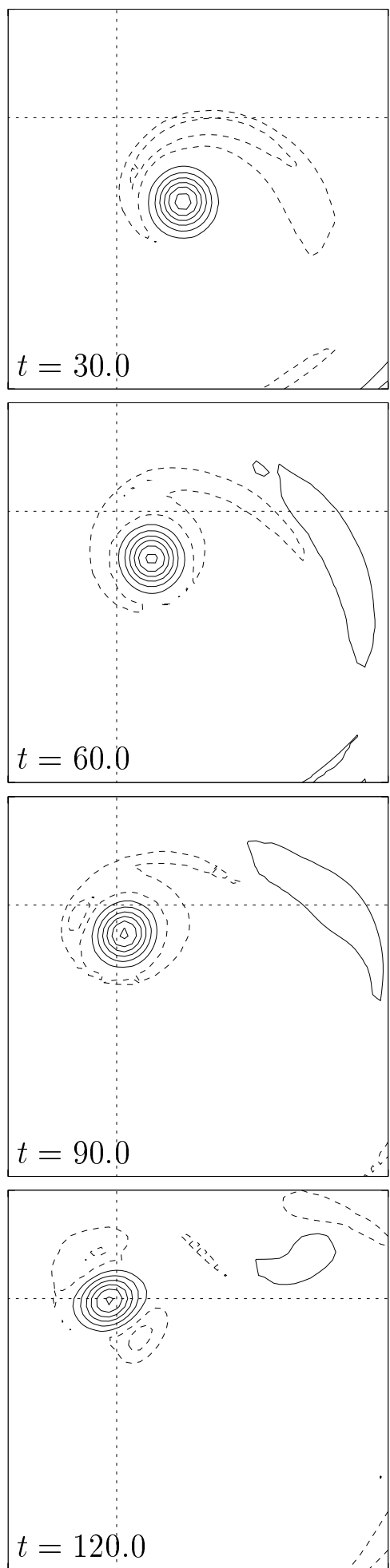

Figure 10: Contour plots of the relative vorticity, as obtained from the relative velocity field, of the vortex of Figure 9 on the $\gamma$-plane at corresponding moments in time. The domain shown is $[-2,5] \times[-5,2]$. 


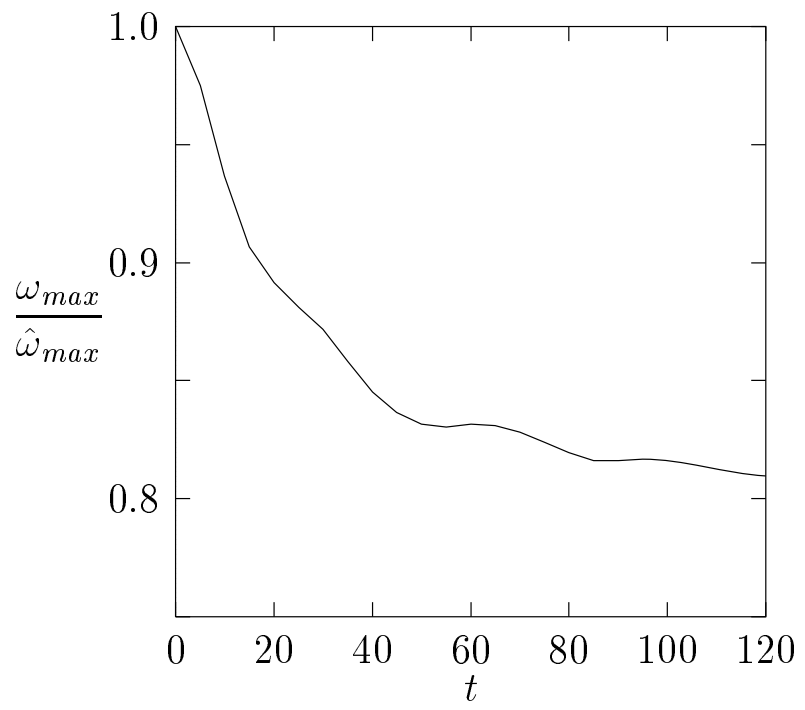

Figure 11: The maximum value of the relative vorticity $\omega_{\max }$ divided by the initial maximum value $\left(\hat{\omega}_{\max }\right)$ as a function of time $t$.

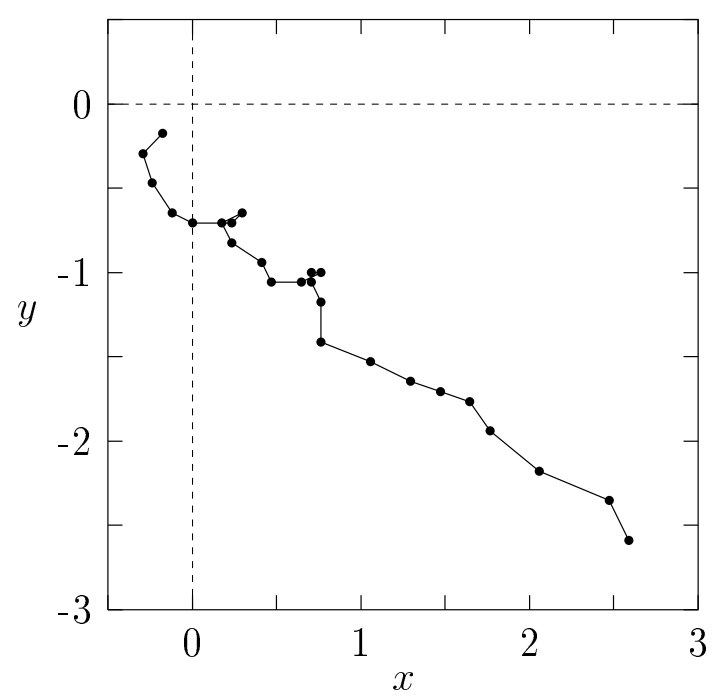

Figure 12: The path of the Lamb monopole. Symbols are placed at the position of the monopole at $t=0,5,10, \ldots, 120$. 


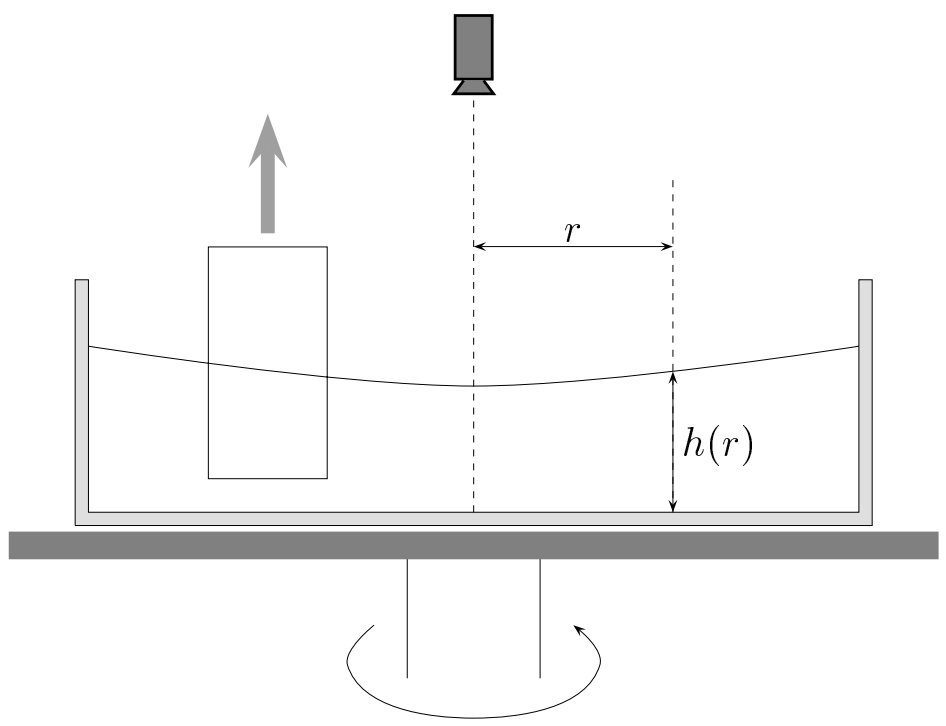

Figure 13: Schematic picture of the experimental set-up. 

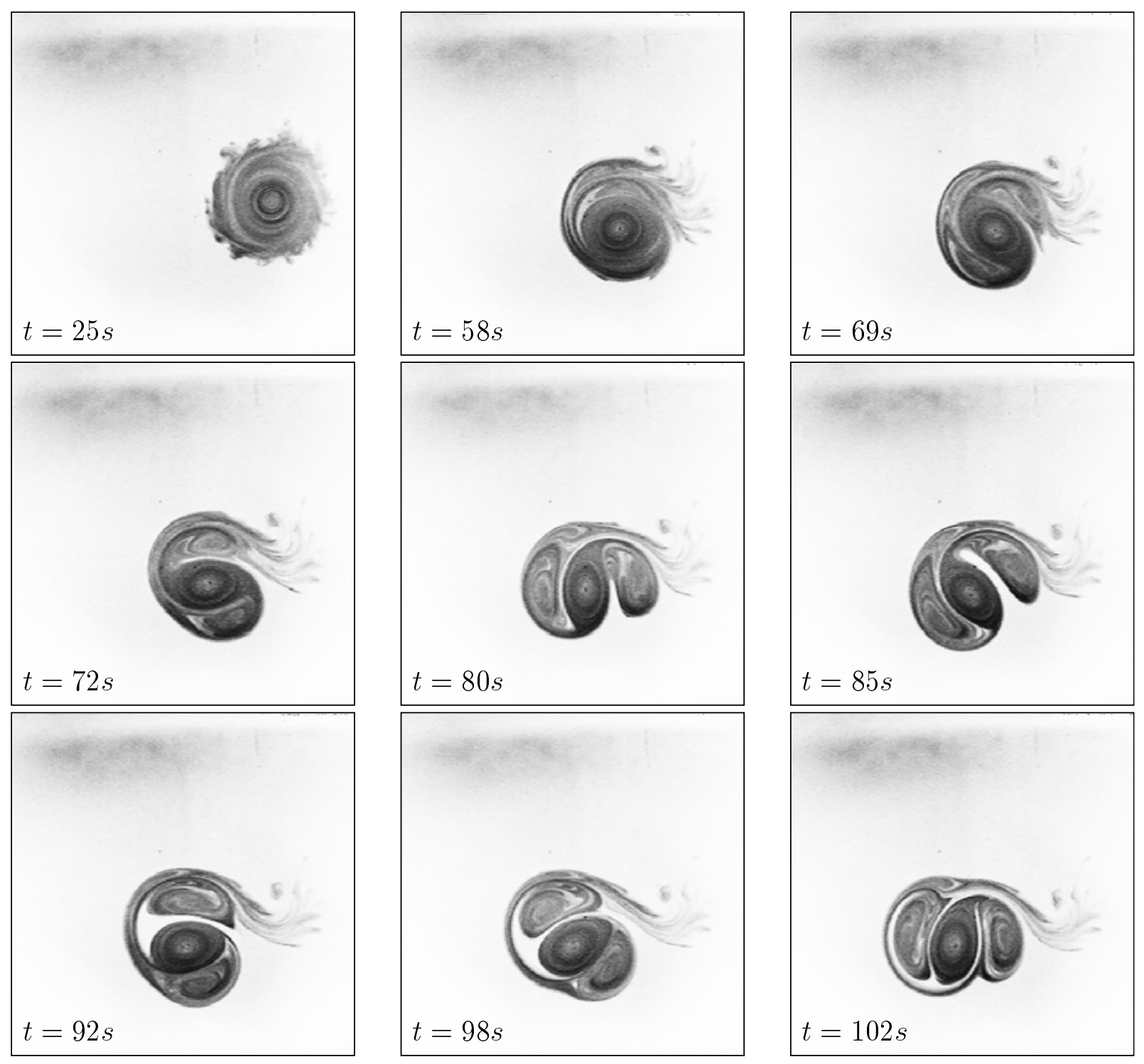

Figure 14: A sequence of images showing the evolution of a tripolar vortex on a topographic $\gamma$-plane. The experimental parameters are $\Omega=0.61 \mathrm{~s}^{-1}, h_{0}=0.16 \mathrm{~m}, \gamma=0.15 \mathrm{~m}^{-2} \mathrm{~s}^{-1}$. The vortex is created approximately $0.25 \mathrm{~m}$ from the rotation axis. 

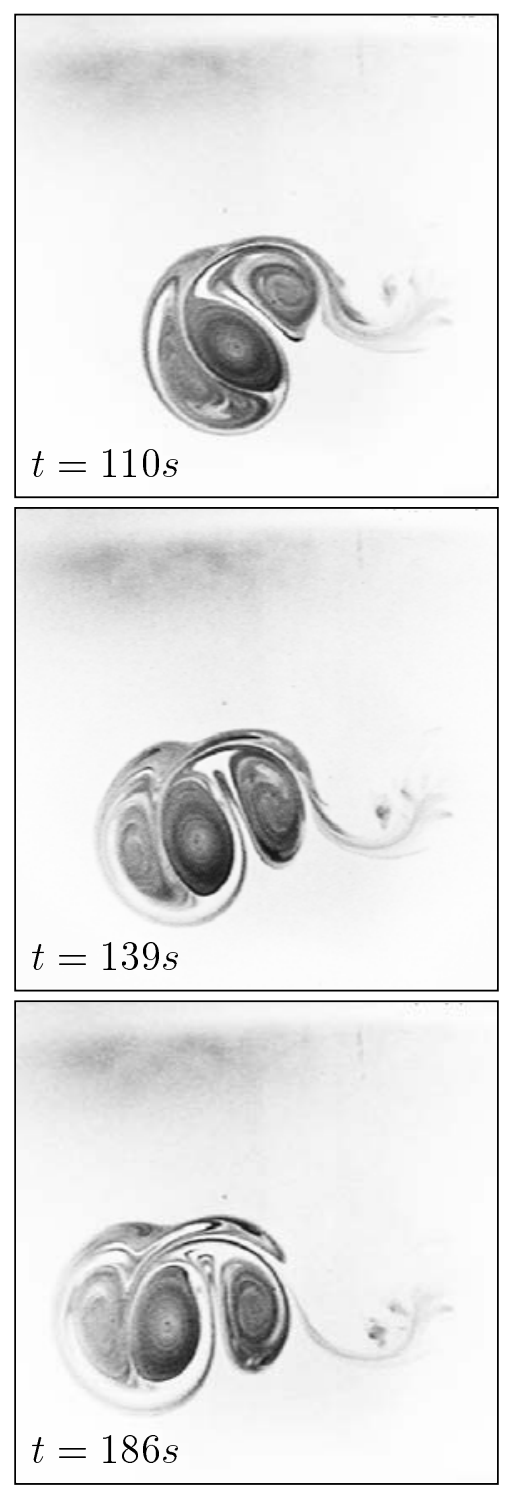
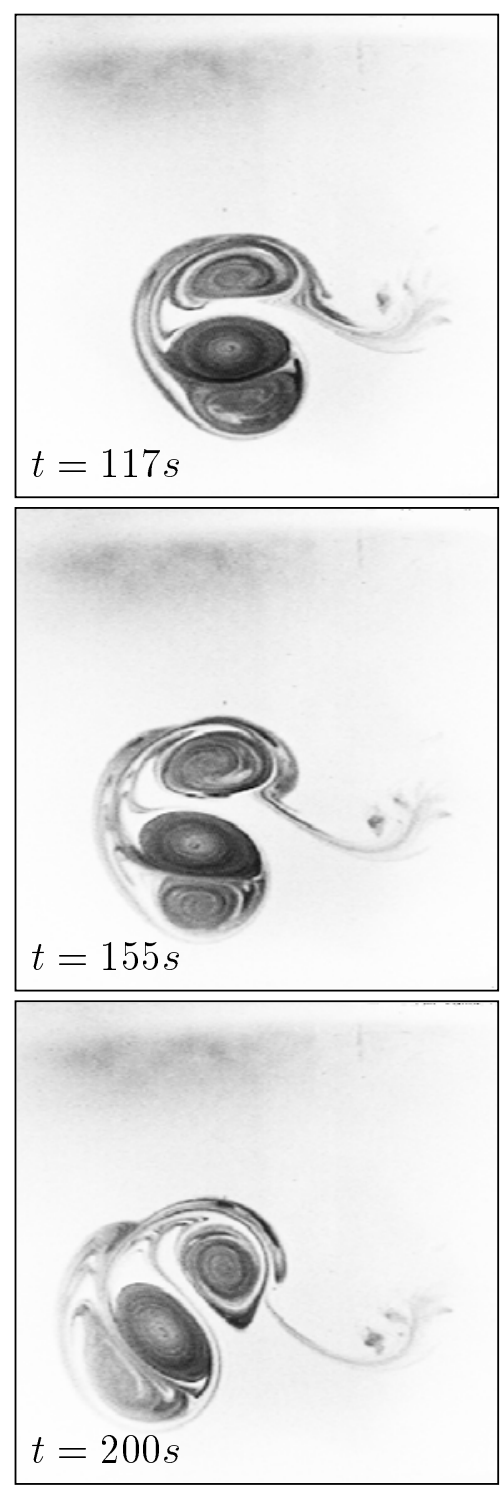
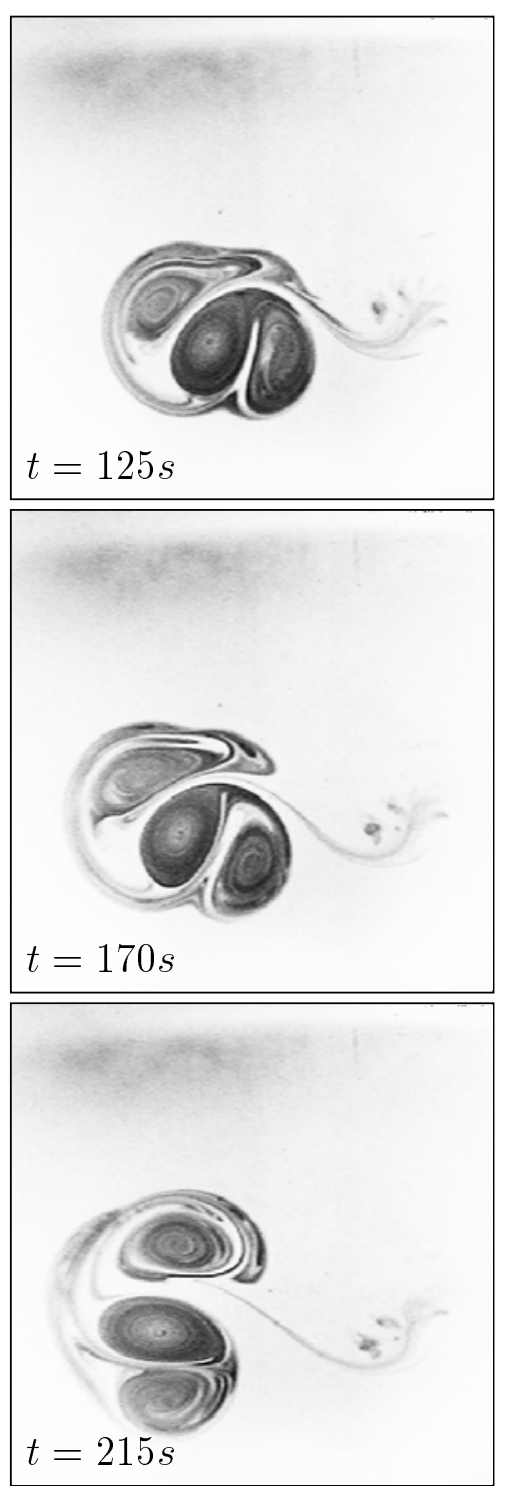

Figure 14 (continued): A sequence of images showing the evolution of a tripolar vortex on a topographic $\gamma$-plane. The experimental parameters are $\Omega=0.61 \mathrm{~s}^{-1}, h_{0}=0.16 \mathrm{~m}$, $\gamma=0.15 m^{-2} s^{-1}$. The vortex is created approximately $0.25 m$ from the rotation axis. 

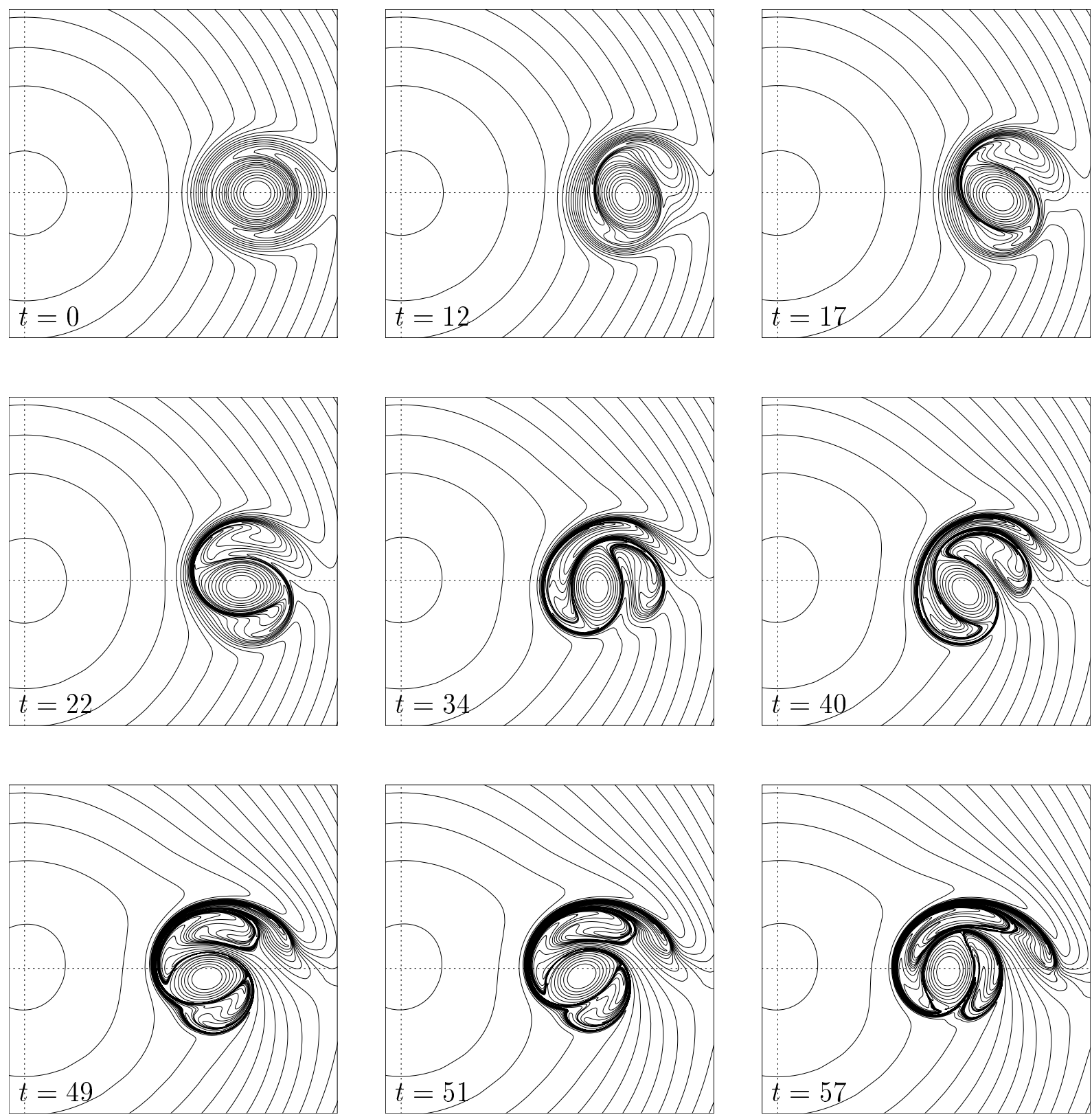

Figure 15: A sequence of pictures showing the evolution of the absolute vorticity contours of the numerical simulation at several stages. The domain shown is $[-0.2,4.0] \times[-1.85,2.35]$. 

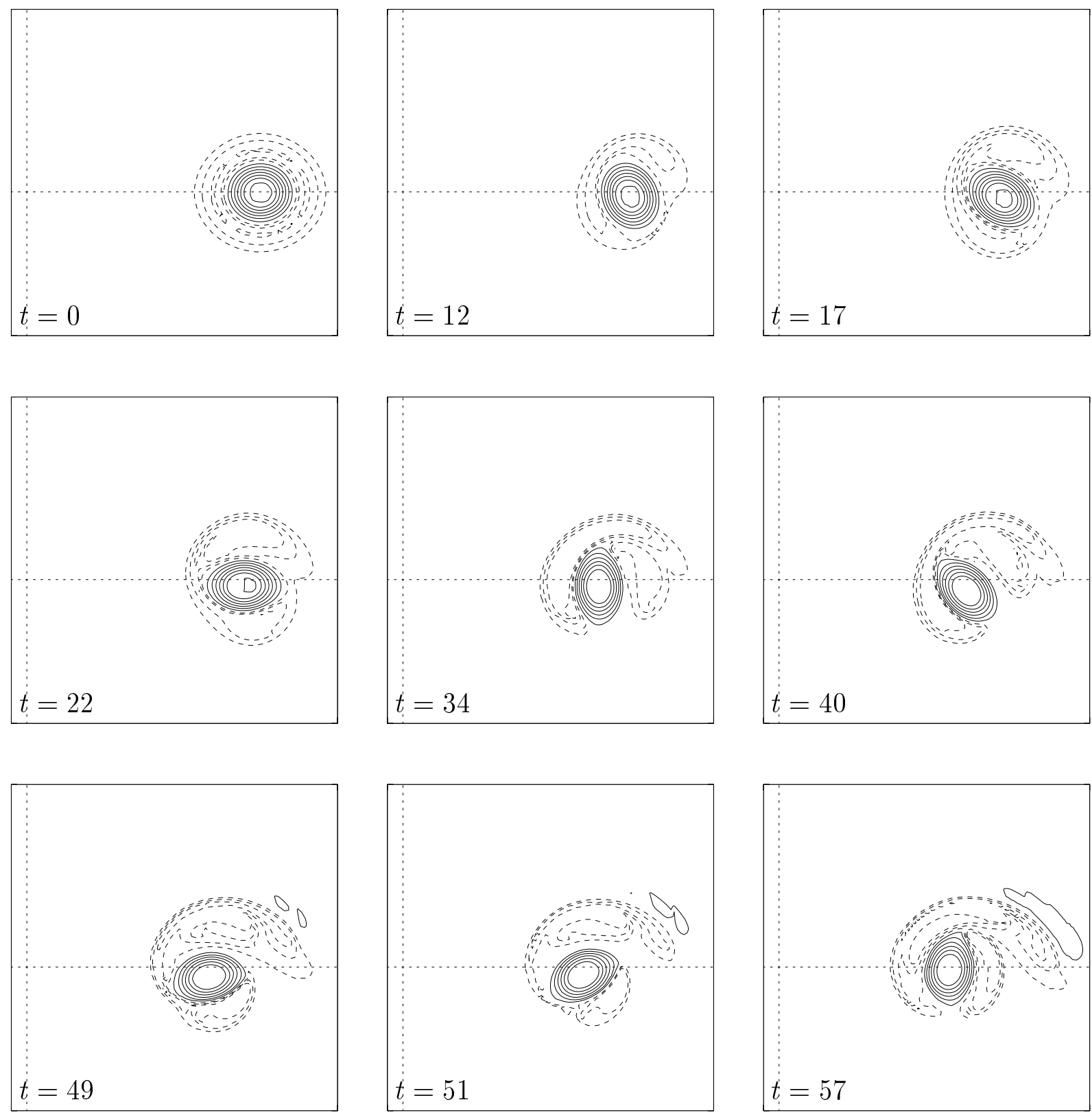

Figure 16: The same as Figure 15 but now the relative vorticity, as obtained from the relative velocity field, is shown. 


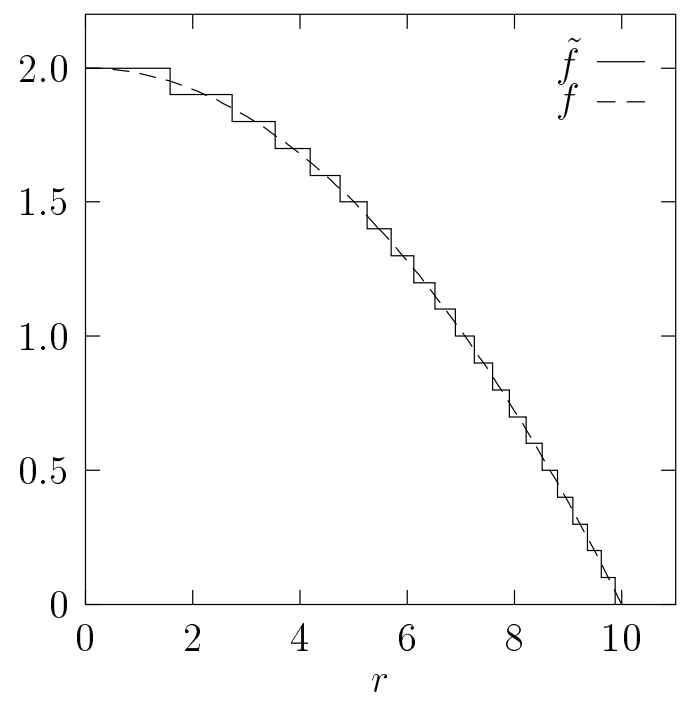

a)

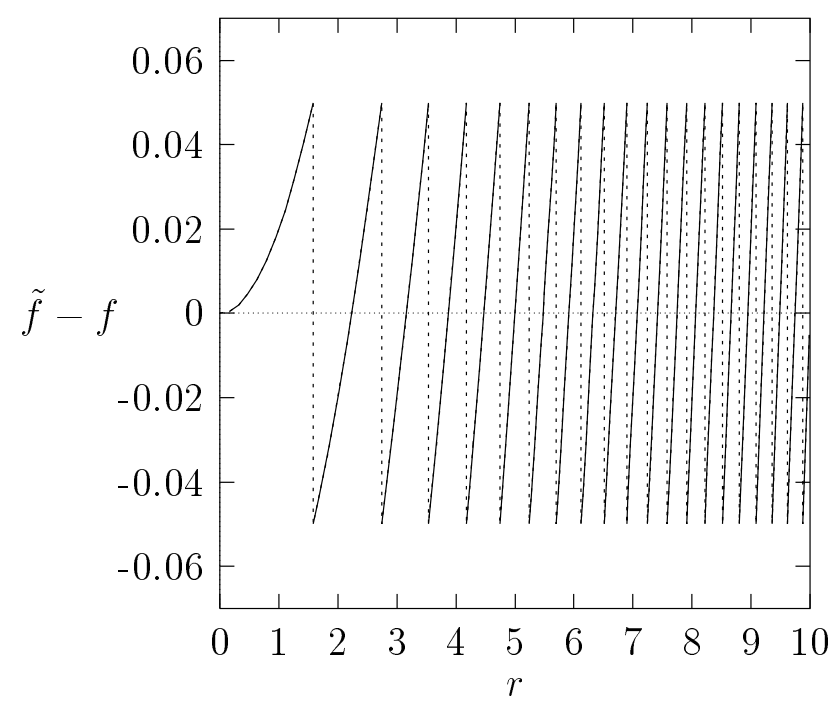

b)

Figure 17: The piecewise-uniform distribution $\tilde{f}$ (solid line) and the continuous profile $f$ (dashed line) in the case of $M=20$ contours, $\gamma=0.02, R_{0}=10$ and $f=\gamma R_{0}^{2}-\gamma r^{2}$ (a) and their difference $\tilde{f}-f(\mathrm{~b})$. 


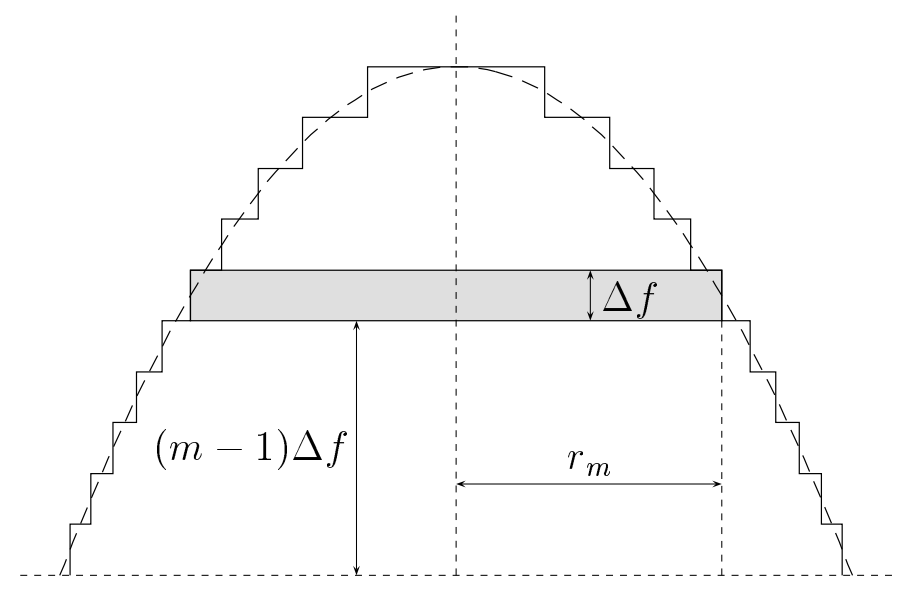

Figure 18: The radius $r_{m}$ is chosen such that the circulation of the grey layer enclosed by the piecewise-uniform profile (solid line) is equal to circulation of the same layer but enclosed by the continuous profile (dashed line). 


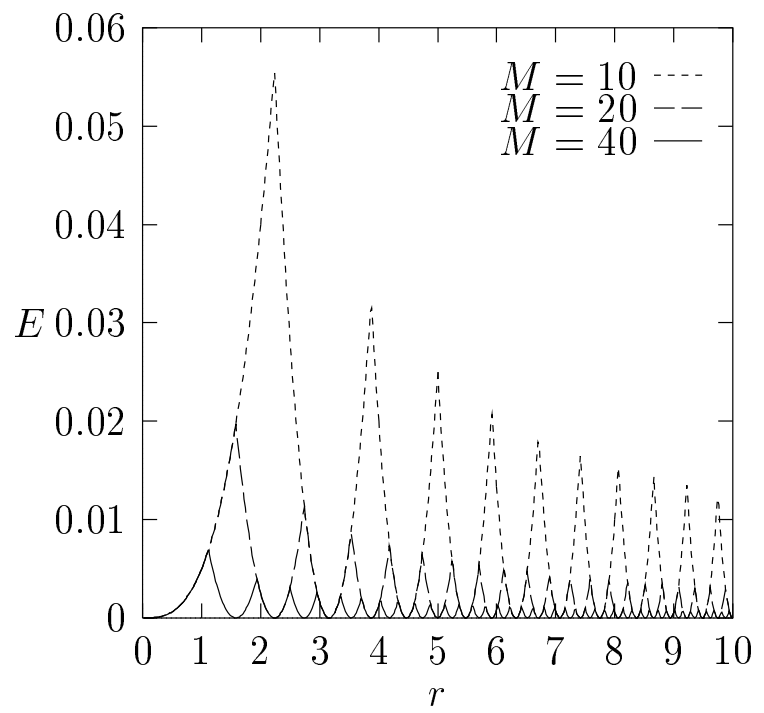

a)

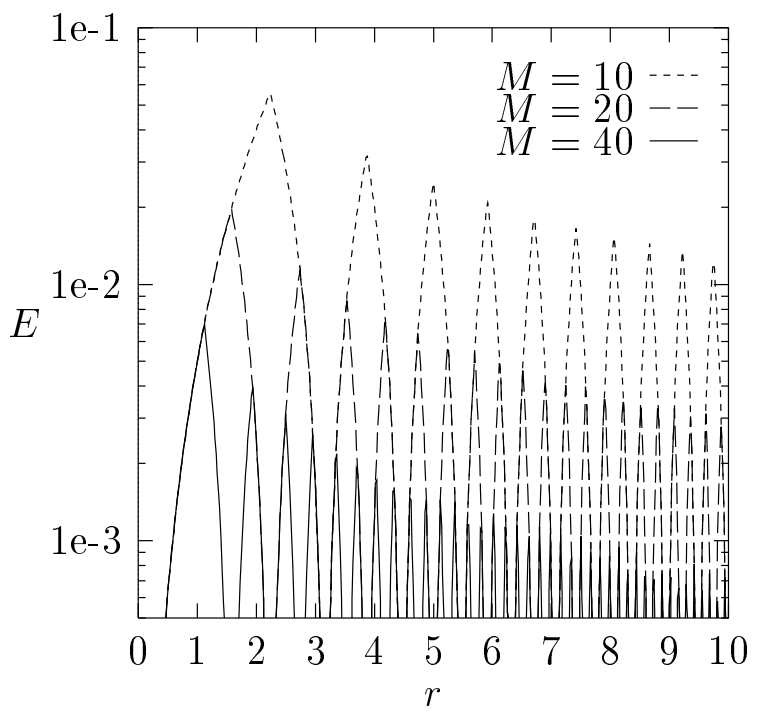

b)

Figure 19: The error $E$ in the azimuthal component of the relative velocity as a function of $r$ on both a linear (a) and a logarithmic scale (b) for several values of $M$ and the same values of $f_{0}, \gamma$ and $R_{0}$ as in Figure 17. 